\title{
Flexible TDMA/WDMA Passive Optical Network: Energy Efficient Next-Generation Optical Access \\ Solution
}

\author{
Abhishek Dixit ${ }^{1}$, Bart Lannoo ${ }^{1}$, Goutam Das ${ }^{2}$, Didier Colle $^{1}$, Mario Pickavet ${ }^{1}$, Piet \\ Demeester $^{1}$ \\ ${ }^{1}$ Ghent University - iMinds, Gaston Crommenlaan 8, B-9050 Ghent, Belgium \\ ${ }^{2}$ IIT kharagpur, Kharagpur, India \\ Phone: +3293314977 \\ Fax: +3293314899 \\ Email: abhishek.dixit@intec.ugent.be \\ Website: http://www.ibcn.intec.ugent.be
}

\begin{abstract}
Access networks must further advance to address the intensification of the requirements of growing speeds and the usage of Internet applications, and time and wavelength division multiple access (TDMA/WDMA) based passive optical networks (TWDM-PONs) have been widely considered as one of the evolutionary steps of next-generation optical access (NGOA) networks. TWDM-PON combines the flexibility of TDMA with an increased capacity offered by the use of a WDM layer. Moreover, it offers interesting and challenging avenues to minimize energy consumption: especially, with current access networks consuming about $80 \%$ of the energy consumed in the Internet. Along with other efforts, reducing energy consumption of central offices is conspicuous as it directly minimizes the operational expenditures of network providers. In this paper, we explore the new paradigms to conserve energy at the central offices in TWDM-PONs. By extensive simulations, we evaluate the possible energy savings in the various flavors of TWDM-PON. Based on the findings, we propose a new architectural flavor of TWDMPON and benchmark the architecture for cost, power consumption and reach. We also propose a novel energy saving scheme for the proposed architecture and evaluate the impact of the proposed algorithm on energy savings by extensive simulations.
\end{abstract}

Keywords: energy efficiency; flexibility; optical access; PON architectures;

TDMA/WDMA PON;

\section{Introduction}

Access Evolution from $x$ DSL to FTTX - xDSL and cable modem technology made improvements in bandwidth capacity but have physical limitations to open the capacity bottleneck that exists in subscriber access networks, which cover the "first mile" areas and serve numerous residential and small business users. Thus, xDSL and cable modem technology are being replaced by optical network technologies, like fiber-to-the-X (or

A short summarized version of this paper was presented at the IEEE Advanced Networks and Telecom Systems (ANTS) 2011 Conference in Bangalore, India, in December 2011. 
FTTX, where X can mean the home, curb, cabinet, or building). FTTX technologies have been envisioned as a preferred solution because of their potential to provide high data rates and low energy per bit to subscribers, and passive optical networks (PONs) have been widely considered as a promising technology for implementing various FTTX solutions. Currently, a variety of PON systems has been proposed, with the most used one being the time-division multiplexing (TDM) based PON. Meanwhile, two variants of TDM-PON, gigabit-capable PON (GPON) [1] and Ethernet PON (EPON) [2], have been used for mass rollouts. GPON is widely deployed in parts of the US and Europe whereas EPON is deployed in Japan and Korea. Further global PON deployment is expected at a compound annual growth rate of $28.67 \%$ [3].

Requirements of a next generation optical access solution -With the ever-increasing requirements of bandwidth, a next generation optical access (NGOA) network with a higher bandwidth per customer is a natural path forward [4]. The NGOA solution should also allow seamless migration of customers and should coexist with legacy GPON and EPON networks to protect the investment of network operators. In addition, network operators require many additional attributes to optimize their investments in the NGOA solution. With this in mind, currently the requirements of an optimal NGOA solution are being actively investigated, e.g., done in the European FP7 project OASE (optical access seamless evolution). In OASE, the following key requirements are defined for an NGOA by 2020 [5]:

- Support of $128 \mathrm{Gbit} / \mathrm{s}$ up to $500 \mathrm{Gbit} / \mathrm{s}$ aggregated capacity per feeder fiber,

- Support of 256 up to 1024 optical network units (ONUs), i.e. customers, per feeder fiber,

- Support of 20 to $40 \mathrm{~km}$ extended passive reach option for the working path,

- Low energy consumption,

- Low capital and operational expenditures,

- Coexistence with GPON.

In general, the requirements aim to facilitate high bandwidth per customer, a high customer fan out, long reach, energy efficiency, while maintaining low cost and coexistence with GPON. It is easy to comprehend the main motivation behind the design requirements of an NGOA solution. Clearly, the requirements of bandwidth will further increase the coming years, and new applications will pop up to consume available network bandwidth. A high customer fan out allows more efficient sharing of fiber, components and rack space of an optical line terminal (OLT) at a central office 
(CO), and thus minimizes the cost and the power consumption of the network per user. Long reach is considered for a higher node consolidation, where a central access node (CAN) replaces many active COs. The reduction in active network sites minimizes operational expenditures $(\mathrm{OpEx})$ of an operator. The requirement of low energy consumption is to minimize operational expenditures and greenhouse gas emissions. Low capital and operational expenditures are the main motivations behind the other requirements and hence remain as one of the key requirements of network operators. Coexistence with GPON network (for Europe, GPON is the main legacy deployment) safeguards network providers' investments in legacy GPON deployment.

How legacy networks address these needs? - Currently deployed EPON or GPON systems are unable to provide the expected residential data rates by the year 2020 . Typically, these PON systems are using a separate wavelength (of 1 or $2.5 \mathrm{Gbps}$ ) for downstream (OLT to ONU) and upstream (ONU to OLT), and both wavelengths are then shared among multiple users (e.g., 16, 32, 64). As users share the same pool of capacity, competition arises and traffic requests cannot be honored because of congestion. The mentioned capacity bottleneck for TDM-PONs was tackled by the standardization activities for the 10G xPON systems (10G EPON and 10G GPON, respectively). The physical access bit rate is pushed to $10 \mathrm{Gbps}$ per wavelength, firstly for the downlink part and secondly in a symmetric offer for the uplink part. Nevertheless, 10G xPON systems fail to meet all the requirements of an NGOA solution: especially, a high upstream and a downstream capacity.

NGOA solutions - To fulfill the requirements of an NGOA solution, several architectural flavors have been investigated. Wavelength division multiplexing PON (WDM-PON) provides a dedicated wavelength per customer and offers the most straightforward way of capacity increase. WDM-PON, however, uses the available spectrum inefficiently as there is no sharing of wavelengths. This inefficiency is removed by time and wavelength division multiplexed PON (TWDM-PON) by utilizing a TDM layer in combination with the WDM technology. TWDM-PON has an increased capacity thanks to the WDM technology, and efficiently shares it among many users using the TDM layer. Thus, it is considered as an important NGOA candidate. TWDMPON is selected as a primary candidate of the NGOA solution [6] by the full service access network (FSAN). Notably, FSAN initiated an effort to investigate NGOA solutions in 2011. 
With the selection of TWDM-PON as a NG-PON solution, a large-scale development of TWDM-PON based system concepts is imperative. Thus, TWDM-PON will serve as a preeminent framework for the evolution of future access networks. There are many ways to design TWDM-PON architecture. The architectural solution can share the resources (i.e. wavelength) in a static manner or can provision a more dynamic sharing of the network resources. The dynamicity of resource sharing is referred as flexibility [7], [8]; in general, there can be two extremes of flexibility: fully flexible and static. For example, in TWDM-PON, the network resource is a set of wavelengths, and if all users can share all wavelengths at all time instances, then the architecture is fully flexible, whereas if all users have access to just one wavelength, then the architecture is inflexible or static. The most common example of a fully flexible architecture is TDMPON; the network resource is one wavelength and all users dynamically share this wavelength.

Flexibility brings with it several advantages. Most importantly, it facilitates bandwidth allocation mapped to the demand and the behavior of users. As a result, it copes with the burstiness of network traffic and minimizes the use of all wavelengths. This enables use of wavelengths at high utilization and switching off unused wavelengths, leading to energy efficiency. While there are advantages of flexibility, there are also constraints like security, power budget, etc. Thus, there is a need to quantify the advantages and the disadvantages of flexibility to design an optimal architecture.

This paper first evaluates the optimal degree of flexibility and based on that proposes an optimal architectural solution. For the proposed architecture, we introduce a novel energy saving mechanism, which reduces significantly the power consumption of OLTs.

The remainder of the paper is organized as follows. In Section 2, we discuss the architectural solutions and system concepts of TWDM-PON that can extract the flexibility advantages. In Section 3, we quantify flexibility and identify the optimal degree of flexibility. In Section 4, we propose a flexible TWDM-PON architecture based on the findings in Section 3, and benchmark its cost, power consumption and reach with the other variants of TWDM-PON. In Section 5, we propose a novel energy saving mechanism for the considered architecture and evaluate its gains through exhaustive simulations. 


\section{TWDM-PON and Approaches to Design Flexible Architectures}

In this section, we first discuss basic TWDM-PON architectures and then discuss the approaches to design flexible architectures.

\subsection{TWDM-PON Architectures}

One of the drivers of the evolution of optical access networks is a higher node consolidation. In a node consolidation scenario, a single CAN replaces multiple active network sites (i.e., local exchange), reducing the operational cost of the network. This drives the OLT to move toward the core, increasing the distances between the OLT and ONUs, as shown in Figure 1. This also allows an NGOA solution to have two or more splitting stages. Thus, in the NGOA scenario, the OLT is located at the CAN and is connected to remote node 1 (RN1) at the local exchange (LE) by the feeder fiber (FF). Through the distribution fiber (DF), each output port of RN1 goes to a different remote node 2 (RN2), and then each output port of RN2 is connected to a different ONU by the last mile fiber (LMF). Moreover, the architectures have a tree topology, with the OLT as the root of the tree and the ONUs at the leaves. There are also some proposals for ring-based architectures. Ring-based architectures can employ a fast protection switching mechanism from synchronous optical network/synchronous digital hierarchy (SONET/SDH) technology, but they require the use of add-drop nodes, which increase the insertion loss, costs, and power consumption. Moreover, a large part of the duct network in developed countries was laid before the appearance of the SONET/SDH ring network topology, and thus ring solutions do not provide short-distance paths between nodes [9]. 


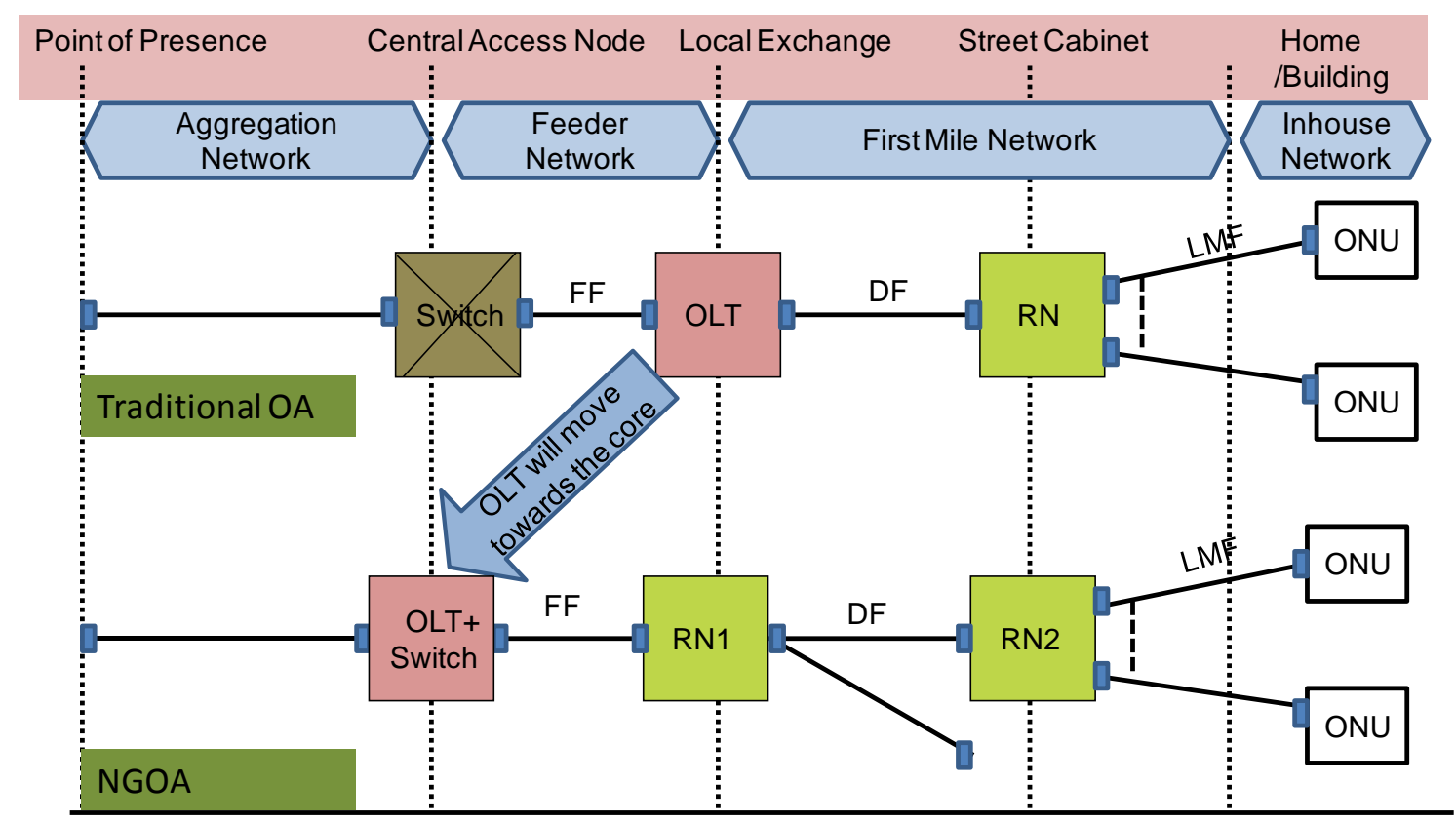

$\mathrm{FF}=$ Feeder fiber, $\mathrm{DF}=$ Distribution fiber, $\mathrm{LMF}=$ Last mile fiber

Figure 1: Difference between traditional optical access (OA) and next-generation optical access (NGOA) scenarios

Figure 2 shows the general architecture of TWDM-PON. The key features of the TWDM-PON architecture are:

- To provide higher peak data rates per user, downstream and upstream channel capacity of $10 \mathrm{Gbps}$ and $2.5 \mathrm{Gbps}$ are considered.

- To support a high fan out (640 or 1280 ), with a sustained data rate higher than $500 \mathrm{Mbps}, 40$ or 80 channels per OLT are considered.

- Migration from deployed optical access networks is one of the key requirements. As RN2 is a power splitter, TWDM-PON uses the already deployed passive optical distribution network (ODN) infrastructure in the field.

Together, with the above-mentioned features, other design criteria, e.g., longer reach, low cost, and low power consumption are desired. We now discuss in detail the system design of the various units of the TWDM-PON:

OLT: Figure 2 shows the detailed system design of the OLT. We use photonic integrated circuit (PIC) based fixed transceivers array (TRXA). The TRXA is designed using PIC to minimize the OLT form factor. Note that we have shown four TRXAs where each TRXA has ten dense WDM (DWDM) channels offering an upstream and a downstream rate of $2.5 \mathrm{Gbps}$ and $10 \mathrm{Gbps}$ per channel, respectively. Each TRXA is composed of ten fixed wavelength lasers. The $\mathrm{C}$ band is used for the upstream and the $\mathrm{L}$ band for the downstream channels, and a series of $\mathrm{L}$ and $\mathrm{C}$ band diplexers are used to multiplex and demultiplex downstream and upstream channels respectively. We 
combine four TRXAs corresponding to 40 upstream and downstream channels (Figure 2). We refer to such a combination of TRXAs as a TRXA bank (TRXAB). The TRXAB seems complex, but serves more customers (640/1280) than the conventional access networks, in which a GPON or an EPON OLT serves only 32 or 64 customers, and thus the cost and the power consumption of TRXAB is not high. Lastly, the erbium doped fiber amplifiers (EDFAs) based pre-amplifier and booster are used to increase the reach. $\boldsymbol{R N 1 :}$ The design of RN1 can vary and governs the flexibility of the architecture. There can be various choices for RN1, e.g., a power splitter, an arrayed waveguide grating (AWG), or an active switch, e.g., wavelength selective switch (WSS). The choice of RN1 is further discussed in Section 2.2 and in Section 4.

RN2: We use a simple power splitter at RN2, easing the migration of TWDM-PON from the deployed GPON (or EPON) architectures.

$\boldsymbol{O N U}$ : Since the cost and the power consumption of the ONUs are not shared, the ONU design should be simple. The transceiver (TRX) design significantly influences cost. To minimize cost and inventory issues, colorless TRX design for ONUs is paramount. Various solutions have been proposed for colorless TRXs, for example, spectrum-sliced light emitting diodes (SSLEDs) [10], amplified spontaneous emission (ASE) spectrumsliced seeded reflective semiconductor optical amplifiers (RSOAs) [11], ASE spectrumsliced injection-locked Fabry-Perot laser diodes (FP-LDs) [12], [13], wavelength reuse with injection-locked FP-LDs [14], modulators with centralized optical carrier generation [15]-[18] and tunable optics [19]. Among the approaches previously listed, the SSLEDs and the RSOAs cannot be modulated at sufficiently high speeds to support the data rates required by the proposed architecture (up to $10 \mathrm{~Gb} / \mathrm{s}$ ), whereas, on the other hand, the directly modulated injection-locked FP-LDs schemes have chirp characteristics that are not suitable for the transmission reach required. The centralized optical carrier generation system requires new fiber connections between the central system and the ONUs, and is thus not able to reduce costs to the point where mass deployment is possible. We propose to use a tunable laser and a tunable filter at the ONUs. Currently, the transceivers are tunable over four nanometers or so, but we expect cheap transceivers that will be tunable over the entire $\mathrm{C} / \mathrm{L}$ band to be available in the next few years. For a further detailed design of the OLT, RN1, RN2 and ONU, we refer the interested readers to [19]. 


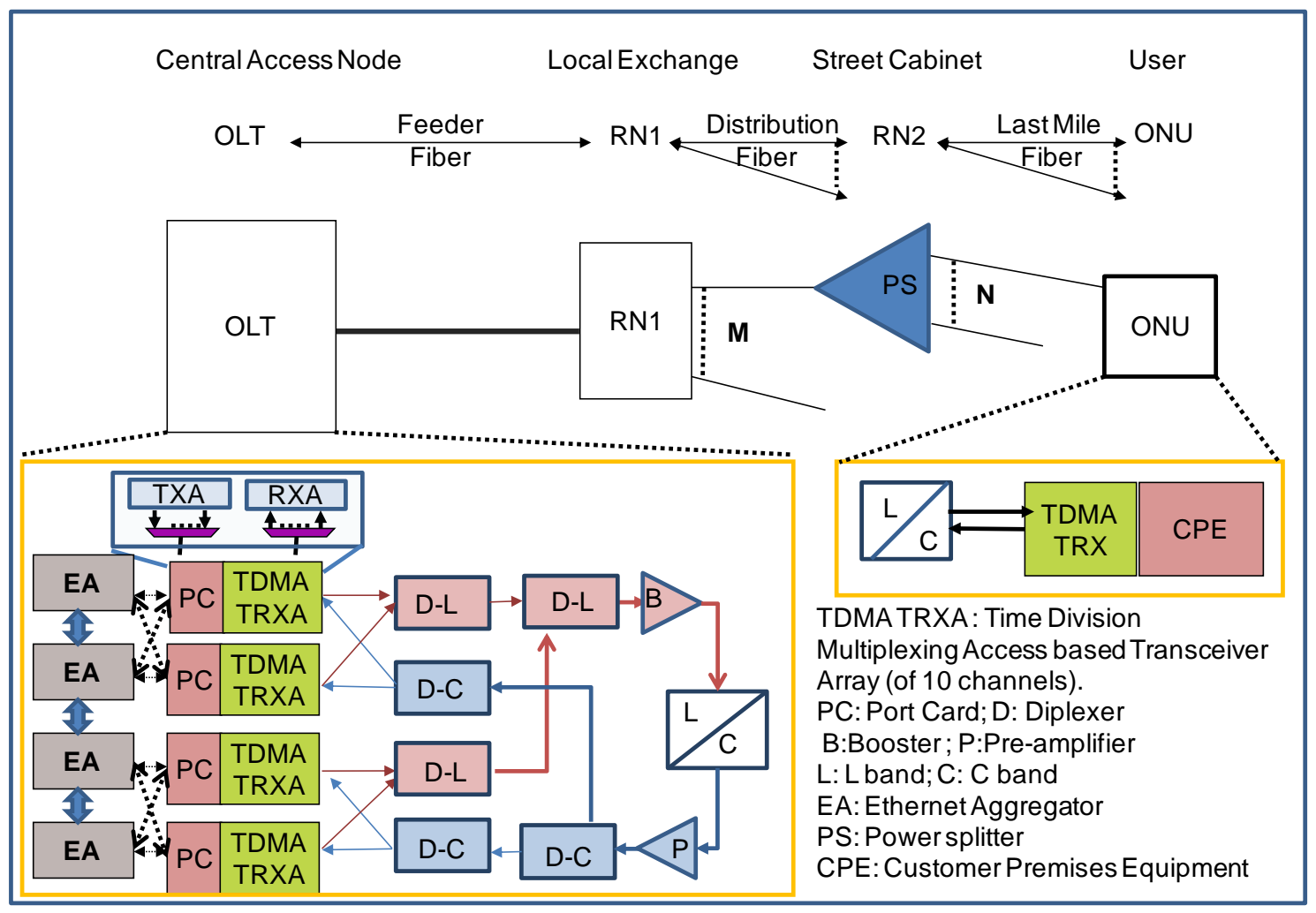

Figure 2: General TWDM-PON architecture, consisting of two remote nodes

\subsection{Design of Flexible Architectures}

By flexible architectures, we mean that the network resources at the OLT should be used efficiently. The network resource that is of importance is the TRXA at the OLT. The TRXAs are used in conjunction with the Ethernet aggregators (EAs), and thus the minimal use of TRXAs minimizes the use of EAs. EAs consume the major portion of energy consumed at the OLT [20]. They account typically for about $14 \%$ of the energy consumed in the Internet, i.e., about $70 \%$ of the energy consumed in the OLT. For resiliency, they are used for normal and backup aggregations.

There are two methods to use effectively the TRXA at the OLT:

1) Flexible $\mathrm{CO}$ design: Provisioning dynamic connection between the OLTs and the PON segments in a CO.

2) Flexible remote node design: Exploiting the remote node design to optimally share the TRXAs among the users in a PON.

\subsubsection{Flexible CO Design}

Currently, a CO serves a large number of customers, and thus has many OLTs. For example, a $\mathrm{CO}$ serves about 16,000 customers in a dense urban scenario. Considering a split of 1:32 as in a conventional GPON, the number of OLTs required per $\mathrm{CO}$ will be 
500. As, however, we consider an OLT serving 640/1280 customers in TWDM-PON, the number of OLTs $(\sim 16)$ per CO will be fewer.

Not all OLTs, however, are always required because of a low data rate or filling ratio. Because of these unused OLTs, there is excessive power consumption at the CO. This excessive power consumption can be reduced by activating the OLTs according to the network load. When the combined network load of the PON segments is low, instead of using an OLT for a PON segment, we share the OLTs. This allows us to shut down the unused OLTs leading to energy efficiency. We now explain this with an example. Let us assume four PON segments per CO. At a low load, e.g. $25 \%$, we are able to serve all four PON segments with just one OLT. This saves about $75 \%$ of the energy consumption. This is the basic idea of a flexible CO design, where we connect OLTs with different PON segments using a flexible optical router (OR), as shown in Figure 3.

The OR combines the OLTs in such a way that they can be used according to the combined network load. The OR broadcasts and selects the wavelengths, and is made up of power splitters and WSSs. The OR has an insertion loss of about $10 \mathrm{~dB}$, which can be compensated using a pre-amplifier (in the upstream direction) and a booster (in the downstream direction) at the OLT. The booster gain at the OLT is limited to keep the total power in the fiber within the laser Class $1 \mathrm{M}$ safety limits. If, however, there is a higher insertion loss within the OLT, the booster gain can be increased to compensate for the additional losses. Thus, insertion losses within the OLT do not affect the reach of the solution. In addition, the power consumption due to the use of WSSs is negligible in comparison to the power savings that can be achieved by shutting down the OLTs.

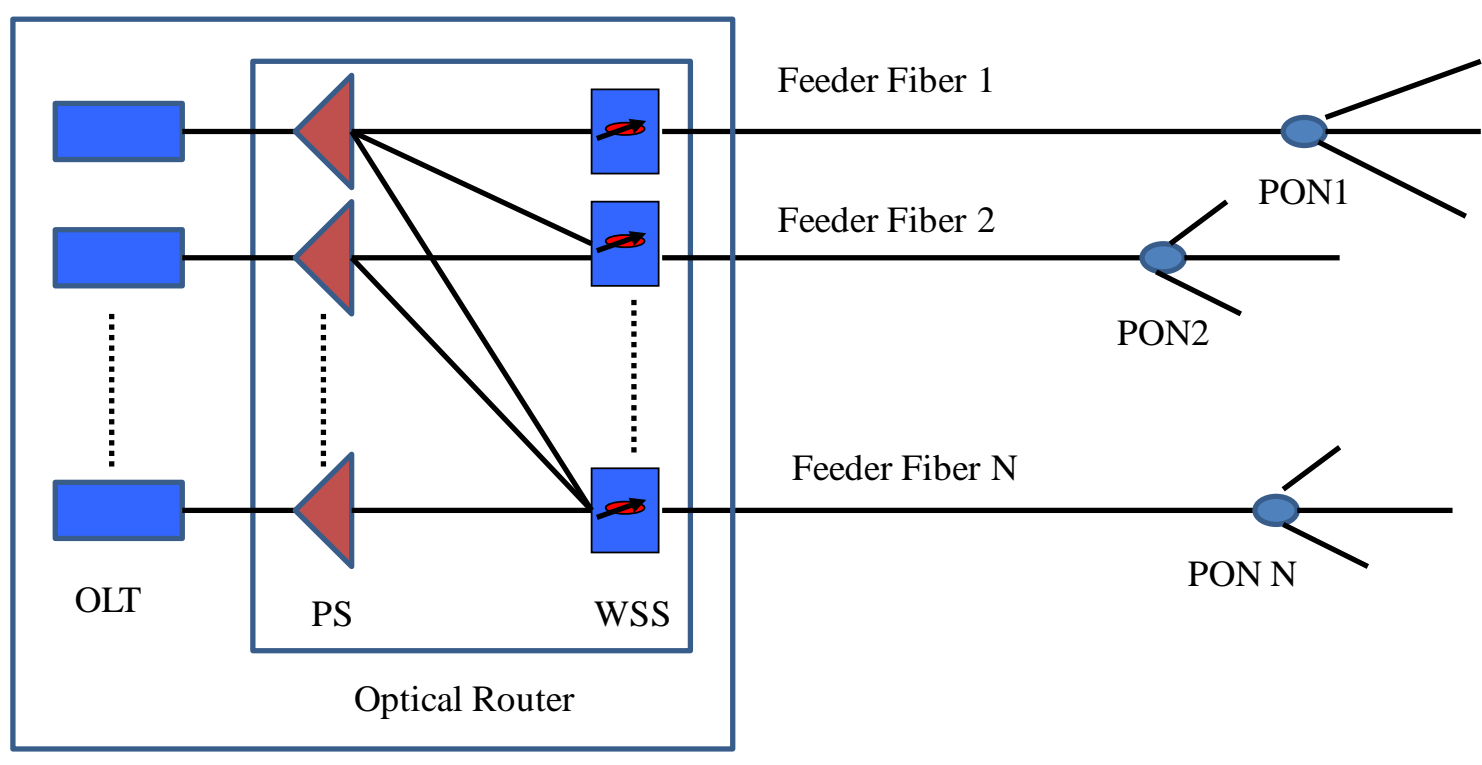

Flexible CO

Figure 3. Flexible CO design to share OLTs with PON segments dynamically. 


\subsubsection{Flexible Remote Node Design}

For a higher flexibility, a flexible $\mathrm{CO}$ can be complemented with flexible remote node (RN1) designs. The straightforward option is to use a power splitter at RN1. The architectures with a power splitter are fully flexible, but have a higher insertion loss, which restricts the reach of the architectures. To maximize the reach of the PON solutions, an AWG is used. An AWG typically has an insertion loss of $4 \mathrm{~dB}$ independent of its fan out. An AWG, however, routes a specific wavelength to a specific output port, and thus the architectures with AWGs are static. To improve the flexibility of the solution with a lower insertion loss, there have been proposals [21], [22] to use WSSs at RN1. The use of WSSs is interesting as it imparts flexibility in resource allocation and has a smaller insertion loss (for 1:8 WSS, the insertion loss is 6 dB) than a power splitter. Based on the RN1 design, the degree of flexibility can thus vary from fully static to fully flexible. We now discuss these various flavors of flexibility:

(1) In a fully flexible architecture, each wavelength is simultaneously routed to all TDM-PONs (or RN2), and by consequence each TDM-PON can be reached by any wavelength. Typical examples of these architectures employ power splitters, and are referred as the wavelength selected TWDM-PON. Figure 4 (a) provides an example of RN1 of a fully flexible architecture. RN1 is a power splitter, and all wavelengths are broadcasted to all TDM-PONs (or RN2s), and by consequence to all ONUs. In this way, each ONU is time shared with the other ONUs with the advantage of having access to a WDM dimension. In the downstream direction, all wavelength channels are broadcasted from the local exchange to all users, without any selectivity in the network itself. The selection is done by the ONU at the user side. This architecture is simple (using a legacy power-split ODN) and provides full flexibility. It, however, suffers from high insertion losses and has a serious security threat as the content of all wavelengths is available to all ONUs. Note that a coherent detection technique at the receiver can deal with the high insertion losses as it allows increasing the optical link budget up to $50 \mathrm{~dB}$ (compared to ca. $30 \mathrm{~dB}$ for direct detection techniques in current PON architectures). Coherent detection, however, is still a complicated and expensive technique to be implemented in practical scenarios for an access network.

(2) In a fully static architecture, each wavelength is routed to only one fixed TDM-PON (or RN2), and each TDM-PON can be reached by only one fixed wavelength. 
Typical examples of these architectures employ AWGs and are referred as the wavelength split TWDM-PON. Figure 4 (b) shows the architecture of a static TWDM-PON. This architecture is also extensively discussed in literature [21] [23]. A cyclic AWG can be assumed to access upstream (e.g., C-band, 1530 - 1565 $\mathrm{nm}$ ) and downstream (e.g., L-band, 1565 - $1625 \mathrm{~nm}$ ) bands [24]. As an AWG has a smaller insertion loss than a power splitter, this architecture has a better power budget and can support more users and a longer reach. Moreover, it also has a high security. Flexibility, however, is restricted as each wavelength is connected to a fixed TDM-PON, and this cannot be rearranged with e.g., a changing traffic demand.

(3) In a partially flexible architecture, each TDM-PON can be reached by multiple wavelengths. Each wavelength can reach either multiple or only one TDM-PON. These architectures are more costly than the fully flexible or the fully static counterparts, but have a higher security and lower insertion loss than the fully flexible architectures, and, of course, are more flexible than a fully static architecture. Often, a trade-off among these different parameters will decide the best architecture in a specific situation. Figure 4 (c) shows an example of a partially flexible architecture in which a partially flexible optical router (PFOR) is used at RN1. There has been an extensive study about the design of PFOR. The passive components based reconfigurable remote node designs are proposed in [25]. The authors of [25] propose passive reconfigurable optical access network (PROAN) in which ONUs are relocated in a subset of two wavelength pairs (each pair contains a downstream and an upstream wavelength). These subsets overlap cyclically in order to enable flexible bandwidth rearrangement. The passive components based reconfigurability, however, imposes a higher insertion loss penalty. The active components based re-configurable solutions are proposed in [26] and [27]. The solution proposed in [26] uses an array of SOAs to provide a fully flexible solution. Because of the use of an array of SOAs, however, the solution will have a tremendous impact on the cost and the energy consumption. The authors in [27] propose a re-configurable remote node configuration based on re-configurable optical add drop multiplexers (OADMs) equipped with micro-resonators. Further, the authors in [28] propose a quasi-passive reconfigurable remote node solution to provide flexibility. The quasi-passive reconfigurable solution consumes power only during reconfiguration and requires no steady-state power. The solutions proposed 
in [27] and [28], however, require a complex remote node element, which is not available off-the-shelf at a low cost. Moreover, many advantages of flexibility can be obtained by using WSSs. WSSs are widely used in core networks, ensuring their large-scale production, which optimizes their cost. Furthermore, because of a largescale research focusing on their design and development, WSSs are expected to be available with a lower cost, a higher fan-out and a lower insertion loss. In addition, currently there are solutions of WSSs that are available with selective multi-casting, which offers interesting vistas to offer flexibility.

There, however, are concerns regarding the use of active components. Nevertheless, the arguments against the use of active elements in a field can be invalidated because of the following reasons. Firstly, as the TWDM-PON has a high fan out, the power consumption and the cost of using an active element per customer is low. Secondly, in NGOA PON, the RN1 is at the present location of a CO where electric power is already available and thus power feeding does not cause additional expenses. Lastly, no PON technology can simultaneously meet the high fan out and reach requirement of NGOA architectures [5], and all solutions thus require at least active reach extenders.

Further, note that, even though a partially flexible solution employs active components, they have been still referred as PON as the technology is a PON derivative and the data remains optically transparent.

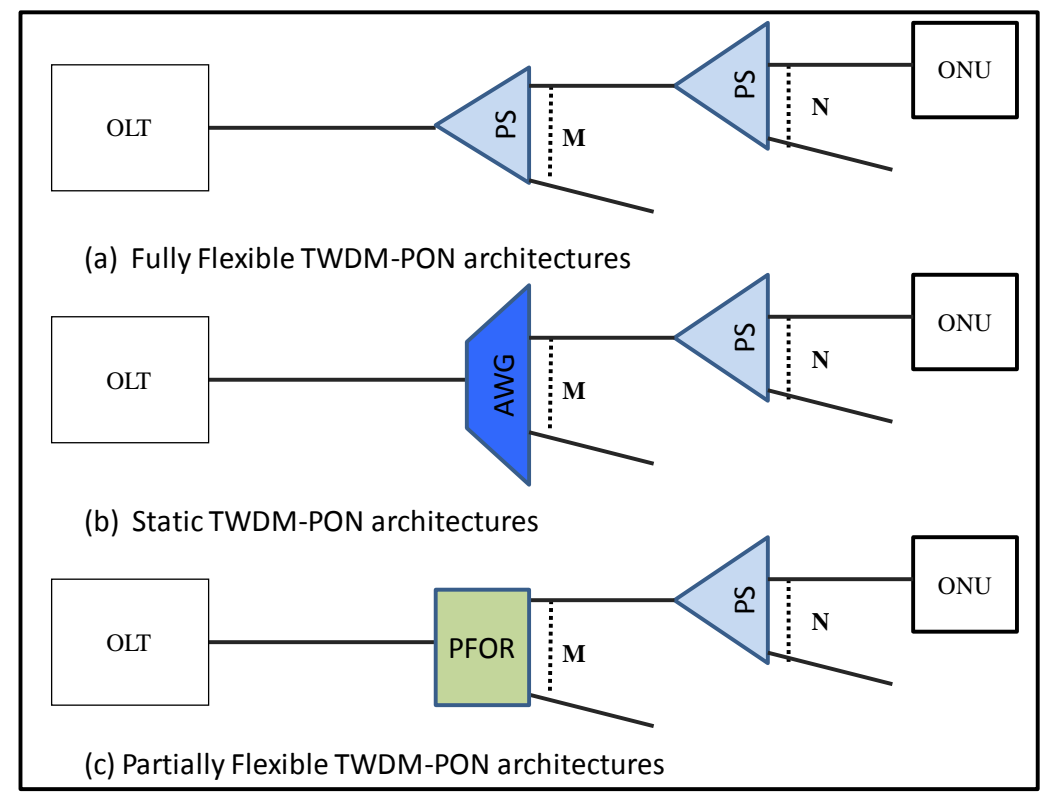

Figure 4: Different variants of TWDM-PON based on the degree of flexibility, where remote node 2 is always a power splitter (PS). 


\section{Evaluation of Flexibility}

In this section, we evaluate the flexibility of various flavors of TWDM-PONs. Papers [7] and [8] have done some preliminary evaluation of flexibility in TWDM-PONs. In this section, we first dicuss the figure of flexibility (Subsection 3.1), and then outline various advantages (Subsection 3.2) and constraints (Subsection 3.3) of flexibility. Following this, we discuss in detail the evaluation approach to investigate flexibility (Subsection 3.4). Finally, simulation results (Subsection 3.5) and conclusions (Subsection 3.6) are discussed.

\subsection{Figure of Flexibility}

There can be many interpretations and meanings of flexibility. As pointed out before, flexibility is the architectural capability where all users dynamically share the network resources (i.e. wavelength). Flexibility is of two types:

\subsubsection{Multicasting Flexibility}

In architectures having multicasting flexibility, a wavelength is shared among many users. Because of this, when the load of the users sharing the same wavelength is small, a new wavelength is not allocated to them. This reduces the channel underutilization, and consecutively the wavelength use, as now one user is necessarily not allocated one wavelength.

Multicasting flexibility stems from using power splitters at remote nodes. Because of a power splitter at RN2, there is always some degree of multicasting flexibility present in a TWDM-PON.

\subsubsection{Switching Flexibility}

In architectures with switching flexibility, the wavelengths are dynamically re-routed according to the instantaneous traffic demand. By dynamic configuration of wavelengths, the users with a high load are allocated more wavelengths, improving the performance. This leads to a smaller probability of the network being overloaded. To measure this probability of overloading, we introduce the network overloading factor (NOF), which is defined as the probability of the network load exceeding the network capacity. A higher NOF leads to higher delays in the network. The architectures with active components, e.g., WSS in RN1, offer switching flexibility. 


\subsection{Advantages of flexibility}

In a TWDM-PON, multiple TDM-PONs can be set up from the OLT, each at a specific wavelength. Each TDM-PON serves a set of users, and within this set, the capacity is shared. By means of wavelength selection or routing, the number of users within the set can be varied, and thus the capacity offered per user can be varied. Hence, a flexible TWDM-PON can offer capacity-on-demand, and the congestion probability can be significantly reduced compared to a static wavelength configuration. Other advantages of a flexible architecture are network extensibility, energy efficiency, and network migration [22], [29].

\subsection{Constraints Raised by Enhanced Flexibility}

Flexibility is added in the network by the use of either active switches or power splitters. While adding active switches increases cost, the use of power splitters increases insertion loss. In addition, power splitters broadcast signals to all users, raising potential security concerns. Thus, adding extra flexibility in the access network comes at the cost of drawbacks like higher equipment costs or higher insertion losses and security issues.

\subsection{Evaluation Approach of Flexibility in TWDM-PONs}

As discussed, TWDM-PONs can have a different degree of flexibility. A question that arises is if a fully flexible architecture is really needed, or if a partially flexible architecture can already serve similar flexibility advantages. In this subsection, we discuss parameters (Subsection 3.4.1), architecture (Subsection 3.4.2), traffic models (Subsection 3.4.3), and medium access control (MAC) protocols and assumptions used for evaluating flexibility.

\subsubsection{Parameters for evaluating flexibility:}

To quantify flexibility, we study two parameters: number of wavelengths used, and the quality of service (QoS) metrics like delay and NOF.

Number of wavelengths used: With access networks consuming about $80 \%$ of the energy consumed in the Internet, energy efficiency is a paramount consideration. For optical access networks, the bulk of the energy is consumed at the ONUs, necessitating research initiatives as in [30], [31]. At the same time, saving energy at the OLT is a key requirement for network operators as it directly influences their operational 
expenditures. An important assessment parameter to quantify energy efficiency is the number of wavelengths required at a certain network load. The minimal use of wavelengths, and consecutively other supporting functionalities like port cards, leads to lower energy consumption and operational expenditures. The number of wavelengths (or transceiver) required is a function of statistical multiplexing of the network: when more users are grouped together on a wavelength, fewer wavelengths are required. To quantify the gains of statistical multiplexing, we will study the average number of wavelengths required vs. the degree of multicasting.

QoS performance: Another parameter that we investigate is the influence of flexibility on the quality of service (QoS) performance. The QoS performance is important to deliver high priority traffic over the network. Average packet delay is one of the important QoS parameters [32]. The delay increases whenever the network gets overloaded. Thus, we study both NOF and queuing delay. For our study, we consider only queuing delay at the ONUs and not the propagation delay between the OLT and an ONU.

\subsubsection{Architecture considered for evaluation:}

We simulate a TWDM-PON with $\mathrm{M}=16$ TDM-PONs, each consisting of $\mathrm{N}=4$ ONUs, and with 16 wavelengths, each with a capacity of 1 Gbps (cf. Figure 2). Note that for the simulations, we do not consider 40 or 80 channels at the OLT as we believe that the gains of multicasting or switching flexibility can be quantified accurately even with a scaled down number of users and wavelengths. We consider five different variations of a partially flexible TWDM-PON, each with a varying degree of flexibility in RN1 by varying the values of $m_{s}$ and $m_{A W G}$ (or $m_{W S S}$ ), while keeping $M=m_{s} \times m_{A W G}$ (or $m_{W S S}$ ) constant (cf. Figure 5). Note that $m_{A W G}$ and $m_{W S S}$ represent the output port number of the AWG and WSS, respectively. For our simulations, we assume the values of $m_{A W G}$ or $m_{W S S}$ as $\{1,2,4,8,16\}$. Not all these configurations of the port numbers of WSS and AWG are available; we, however, choose these values to study flexibility in the most logical incremental steps. For the different variants, Group $x$ indicates the number of TDM-PONs $\left(x=m_{s}\right)$ that can share the same wavelength, or the number of wavelengths that can be used by one TDM-PON (or RN2). In two extreme cases, this architecture is reduced to a fully flexible architecture $\left(m_{s}=16\right.$ or Group 16) and a fully static wavelength-split architecture ( $m_{s}=1$ or Group 1), respectively. The other architectures 
then represent the partially flexible architectures and specify that only 8,4 or 2 specific wavelengths (Group 8, 4, 2) can be used by one TDM-PON.

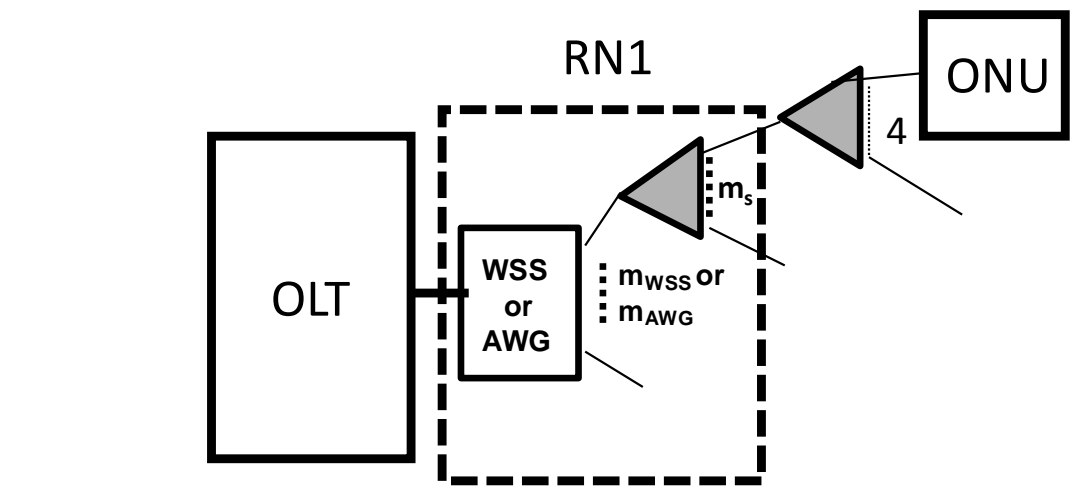

Figure 5: TWDM-PON flavour based on AWG and power splitters considered for evaluation

\subsubsection{Traffic model:}

We study the performance of various variants of flexible TWDM-PON architecture by using the OPNET simulation tool. In our model, we consider $\mathrm{R}_{\mathrm{D}}$ to be the data rate of the access link from a user to an ONU, and $R_{U}$ to be the data rate of an upstream channel from an ONU to the OLT. The maximum distance between the OLT and ONUs is $100 \mathrm{~km}$. We choose $\mathrm{R}_{\mathrm{U}}=1 \mathrm{~Gb} / \mathrm{s}$ and $\mathrm{R}_{\mathrm{D}}=\mathrm{R}_{\mathrm{U}} / \mathrm{N}=250 \mathrm{Mb} / \mathrm{s}$. We generate packets in the form of Ethernet frames (64 to1518 bytes) and packets arrive at each ONU from the end user. The simulated user traffic is self-similar by aggregating $\mathrm{S}=32$ sub-streams [33], each consisting of alternating Pareto-distributed on/off periods, with a shape parameter of 1.4 for the on period and a shape parameter of 1.2 for the off period. During the on period, the packet arrivals are exponentially distributed with a mean arrival rate of $A_{r}$ (in $\mathrm{b} / \mathrm{s}$ ). The variable traffic load can be produced by varying $A_{r}$ and the location parameter for the on and off period. The load is normalized with the maximum upstream capacity $(16 \times 1 \mathrm{Gbps})$.

The reach of the PON also influences its traffic characteristics. A PON with long reach aggregates many users with different traffic profiles (such as business and home users) and over different geographical areas (such as rural and urban). The aggregation of users with different traffic profiles makes the traffic asymmetric. For example, the peak traffic hours of business users are at morning (near $10 \mathrm{am}$ ) and home users are at night (near $9 \mathrm{pm}$ ), leading to an asymmetric traffic. As the aggregation of self-similar traffic and asymmetricity increases burstiness [34], a long reach PON has more bursty traffic. On the other hand, a short reach PON has more symmetric and less bursty traffic. To understand the effect of the traffic on the flexibility evaluation, we produce highly bursty and less bursty traffic. To compare only the effect of burstiness of traffic, we, 
however, use the same reach $(100 \mathrm{~km})$ and the number of users (64) for both less bursty and highly bursty traffic.

Different levels of burstiness can be achieved by varying $A_{r}$. To produce highly bursty traffic, we use an $A_{r}$ value of $12.5 \mathrm{Mb} / \mathrm{s}$ for all loads. To produce less bursty traffic, we vary $A_{r}$ as $0.009 \Phi$, where $\Phi(\mathrm{Mb} / \mathrm{s})$ is the TDM-PON Load. Note that for less bursty traffic, on average, an ONU is switched on for a longer time.

\subsubsection{MAC protocol and related assumptions:}

For evaluating different architectural options of a TWDM-PON, a suitable medium access control (MAC) protocol is needed to manage the time and the wavelength allocation. There exist two approaches of upstream scheduling and wavelength assignment (USWA): online and offline. In the online approach, upon the arrival and processing of a report from an ONU, the OLT immediately decides on the USWA for the corresponding grant. In the offline approach, the OLT waits until it has received all the reports from the ONUs (or part of them) and then it performs some algorithm to find the best USWA scheme for the corresponding grants. The offline approach has a low complexity and can address fairness and QoS issues among different ONUs. Thus, for this paper, we have used an offline scheme based MAC protocol, comparable to the protocol proposed in [35], to investigate the optimal degree of flexibility in TWDMPON architectures.

At the start of a new frame, we compute the number of wavelengths according to the offered load of the ONUs. More concretely, the number of wavelengths for the AWG $\left(N_{A}\right)$ and the WSS $\left(N_{W}\right)$ based RN1 is computed as:

$$
\begin{aligned}
& N_{A}=\sum_{i=1}^{M / G_{N}}\left\lfloor\operatorname{Max}\left(\Lambda_{G} / C, \frac{N_{M} G_{N}}{M}\right)\right\rfloor \\
& N_{W}=\left\lfloor\operatorname{Max}\left(\sum_{i=1}^{M / G_{N}} \Lambda_{G} / C, N_{M}\right)\right\rfloor
\end{aligned}
$$

where $\Lambda_{G}$ is the total aggregate capacity of a group, $C$ is the capacity of a wavelength, $N_{M}$ is the maximum number of wavelengths that can be allocated, $G_{N}$ is the group number, $M$ is the total fan out of RN1, and $\lfloor x\rfloor$ represents the integer greater than $\mathrm{x}$. In an AWG based RN1, the number of wavelengths allocated to a TDM-PON group does not exceed the maximum capacity per group. This condition is relaxed in a WSS based RN1. Note that we assume burst-by-burst switching (neglecting the tuning and the switching time) of the WSS based configuration. Currently, off-the-shelf WSSs are 
available with a switching speed of $250 \mathrm{~ms}$ and thus cannot be used to do burst-by-burst switching. Nevertheless, there have been proposals of the WSSs that can do fast switching in the order of nanoseconds. For example, the authors in [36] propose a wavelength flexible monolithic scheme for fast nanosecond-speed, on-the-fly reconfiguration using monolithically integrated label readers and channel selectors. Currently they are not commercially available and can be extremely expensive for deployment in access networks. However, in the years to come, on-the-fly reconfigurable WSSs with a low cost are expected.

Moreover, the performance degradation due to the tuning and the switching time of the components can be handled by a well-suited MAC protocol, but this is out of the scope of this paper. We have neglected the tuning and the switching time of all components. Paper [37] discusses some of the ways to alleviate the performance degradation due to the tuning and the switching time of the components.

\subsection{Simulation Results}

As already mentioned, we have simulated five scenarios corresponding to different values of $m_{A W G}$ and $m_{s}$. We first study the average number of wavelengths required as a function of the TDM-PON load for different group numbers. This study helps to understand the advantage of grouping users and the benefits of multicasting. We also show the impact of using a static vs. flexible router, e.g., an AWG vs. a WSS, on the average number of wavelengths required. Obviously, the WSS based RN1 should have an advantage over the AWG based RN1 because of its ability to dynamically distribute wavelengths.

We also study NOF and packet delay. We study the overloading condition in an AWG and a WSS based RN1 with different RN2 configurations. This study helps to understand the advantage of switching and multicasting flexibility.

First, we show the simulation results for a TWDM-PON with less bursty traffic and then with supposedly highly bursty traffic.

\subsubsection{Less Bursty traffic}

We first show the average number of wavelengths required as a function of the TDMPON load for different group numbers. As already mentioned, the importance of such a graph is to show how many wavelength line cards are needed. Figure 6 (a) and Figure 6 (b) show the average number of wavelengths required to satisfy the offered TDM-PON 
(or RN2) load in the five considered scenarios in the AWG and the WSS based RN1 respectively. First, we show that from the moment a certain degree of flexibility is available, large gains in wavelength usage are possible, but from a given point the extra gain is limited (the average number of wavelengths used from Group 4 to Group 16 is nearly the same). For example, the Group 2 already minimizes the number of wavelengths used largely as compared to a static solution. The gains of increasing the multicasting beyond Group 2 are less significant, however. We also see that, WSS based RN1 requires fewer wavelengths than an AWG based RN1, as WSS can reroute the wavelengths from one part of the network to the other part and does not need new wavelengths every time the load for a TDM-PON increases.

We then evaluate NOF. Whenever the demands increase more than the resources (which are in the present case 16 wavelengths), the overloading occurs. Figure 6 (c) gives the probability of an overloaded situation in an AWG and a WSS based RN1, expressed as NOF. We show that as the group number increases, the probability of an overloaded situation decreases, since in a larger group base we have flexibility to multicast more wavelengths to each TDM-PON. The WSS based TWDM-PON architectures, however, do not improve the NOF significantly. As the traffic is less bursty, the demands of different groups are nearly the same, and thus dynamic wavelength allocation does not give any clear benefit. Whenever there is an overloaded situation, the delay of the system will increase. Because of the same network overloading condition, both WSS and AWG based TWDM-PON architectures will also have the same delay performance (cf. Figure 6 (d)). The lower bound of the delay equals $1.5 \mathrm{~ms}$ for the considered reach of $100 \mathrm{~km}$ (i.e. $3 / 2$ of the cycle time [38] or $3 / 2$ of the maximum round-trip time of the PON). The simulated traffic has a low peak-toaverage load ratio of approximately 1.15 [i.e. $(\mathrm{Ar} \times \mathrm{N} \times \mathrm{S}) / \Phi=0.009 \times 4 \times 32$ ], and thus the delay is not high even at a high load. 


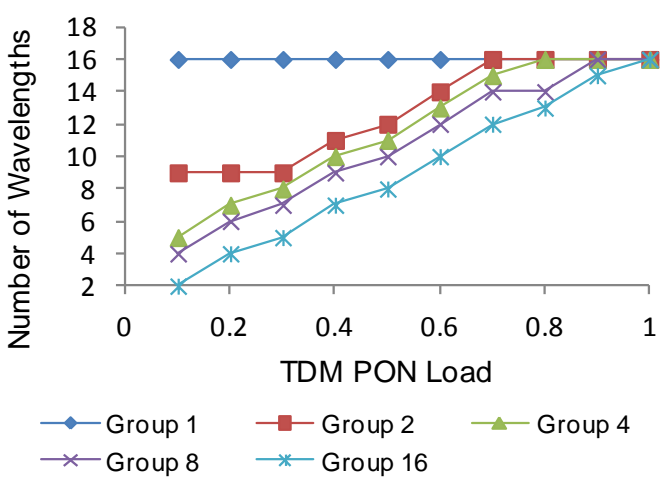

(a)

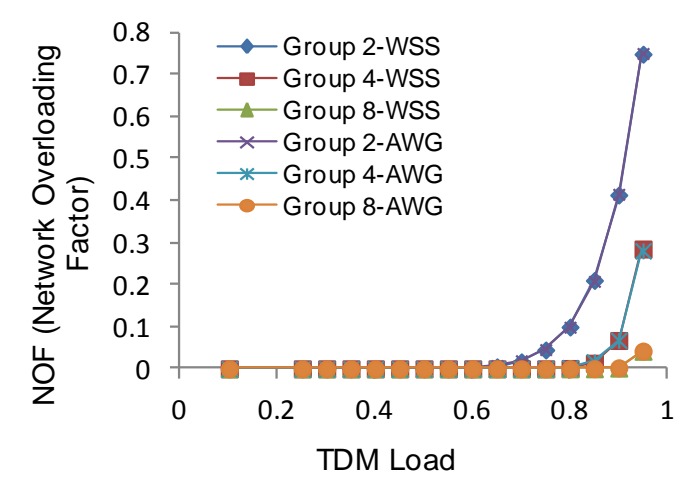

(c)

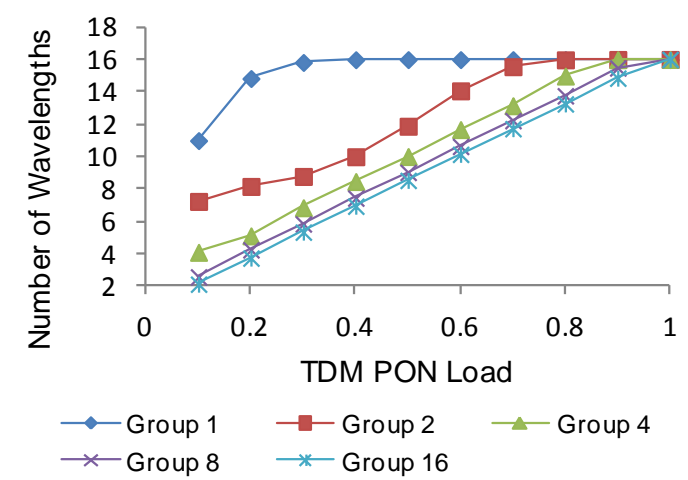

(b)

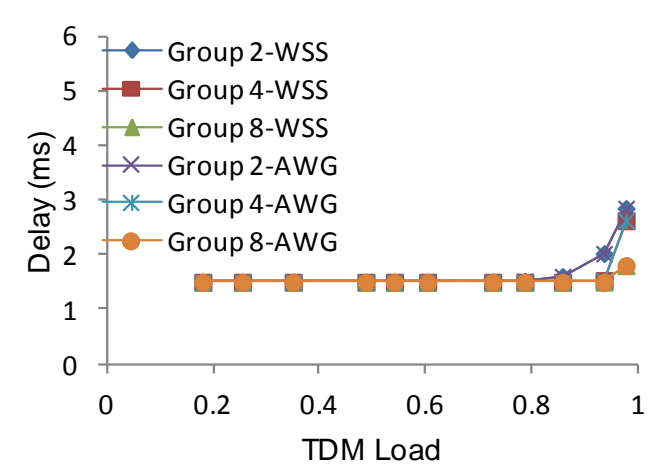

(d)

Figure 6: Illustrates the performance for five TWDM-PON variants with a different degree of flexibility in RN1 for less bursty traffic in (a) Average number of wavelengths required in function of the TDMPON or RN2 load in an AWG based RN1, (b) Average number of wavelengths required in function of the offered TDM-PON or RN2 load in a WSS based RN1 (c) Probability of an overloaded situation in a TDM-PON for varying load for WSS and AWG based configuration, (d) Delay vs. load for TWDM-PON variants with a different degree of flexibility in WSS and AWG based RN1 configuration

\subsubsection{Highly Bursty traffic}

In this section, we show the simulation results for highly bursty traffic, which is mainly noticed in long reach PONs. Figure 7 (a) and Figure 7 (b) show the average number of wavelengths required to satisfy the offered TDM-PON (or RN2) load in the AWG and WSS based RN1. There are many notable differences in the number of wavelengths required for highly bursty and less bursty traffic. First, in an AWG based RN1, the number of wavelengths required for all groups saturates much earlier to the maximum value (16) as compared to the case when the traffic is less bursty. Secondly, for a WSS based RN1, the average number of wavelengths required for all groups is almost the same in contrast to the less bursty traffic, where there is a significant difference in the number of wavelengths required for Group 1 (on an average three wavelengths) and Group 16 (on an average 15 wavelengths). This can be explained as in the bursty traffic, a user is "ON" for a smaller period and thus the probability of two users to be "ON" at the same time will be even less. Thus, at the low load condition, when the probability of 
a user to be in active state is less, grouping of users will not make any difference in the wavelength consumption as most of the time only one user of the group will be in "ON" state. If, however, the probability of a user to be "ON" is high (at high load), multicasting has an impact.

Then, we investigate the influence of using a WSS and an AWG based RN1 on the network performance. Figure 7 (c) gives the probability of an overloaded situation for both RN1 configurations. We can clearly see that the TWDM-PON employing WSS at RN1 is less overloaded as compared to the static TWDM-PON with AWG at RN1. When the traffic is bursty, the user demands vary significantly in a group and a WSS can dynamically allocate wavelengths according to the instantaneous group requirements. Thus, for a more bursty traffic, the dynamic wavelength configuration possible with WSS provides significant advantages. The configuration with WSS and Group 2 has less overloading probability than the configuration with an AWG and Group 4. From these results, we show that we can improve the flexibility of PON by adding an active remote node even with reduced power splitting. Moreover, lower values of NOF lead to lower delay values as can be seen in Figure 7 (d). From the above results, it is clear that as the next-generation PONs aim for a higher reach, the WSS based configurations can give significant flexibility advantages. The availability of 1:23 port WSS with a low insertion loss [39] makes it even more promising for the next-generation PON solutions. 


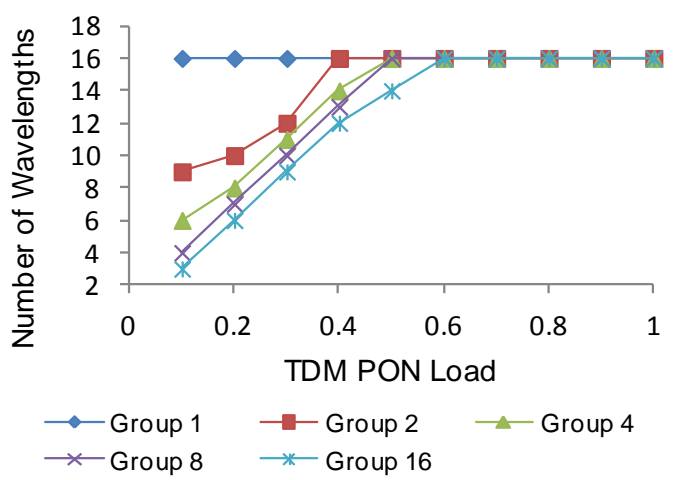

(a)

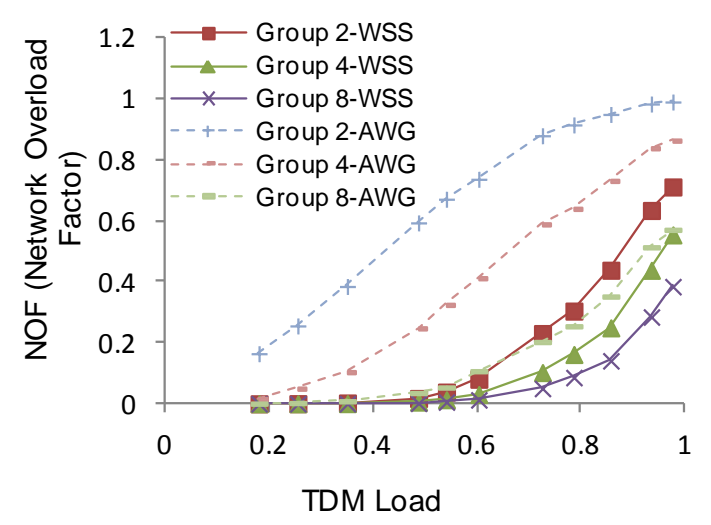

(c)

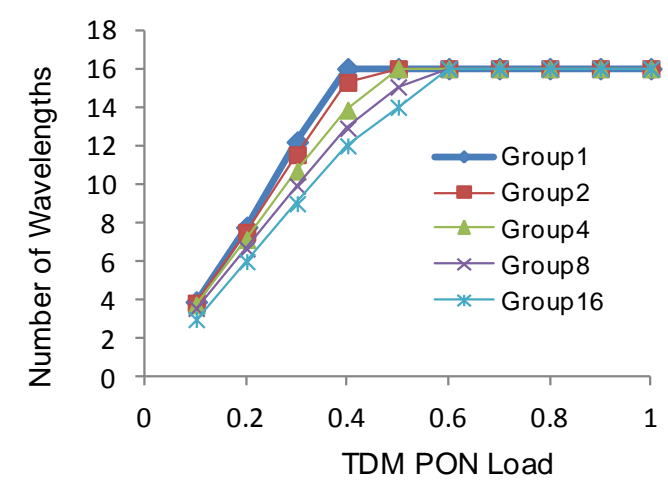

(b)

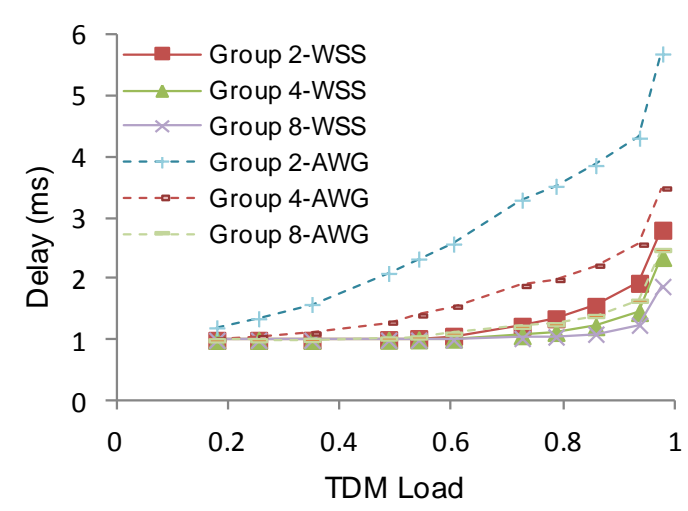

(d)

Figure 7: Illustrates the performance for five TWDM-PON variants with a different degree of flexibility in RN1 for highly bursty traffic in (a) Average number of wavelengths required in function of the TDMPON or RN2 load in an AWG based RN1, (b) Average number of wavelengths required in function of the offered TDM-PON or RN2 load in a WSS based RN1, (c) Probability of an overloaded situation in a TDM-PON for varying load for WSS and AWG based configuration, (d) Delay vs. load for TWDM-PON variants with a different degree of flexibility in WSS and AWG based RN1 configuration

\subsection{Conclusion}

In this section, we have evaluated through simulation the gains of using flexible architectures in next-generation optical access (NGOA) networks, and more specifically in TWDM-PONs. In particular, we have evaluated two types of flexibility gains, due to multicasting and switching. We conclude that a partially flexible network can already provide enough multicasting gain compared to a fully flexible network. Moreover, we have shown through simulations that in long reach PON in which the traffic can be highly bursty, a partially flexible network further benefits from the switching flexibility, which can be delivered by a reconfigurable switch like WSSs. For short reach PON solutions, however, because of presumably less bursty traffic, switching wavelengths does not lead to any significant advantages in PON performance. Thus, if an operator is aiming for higher reaches, then using a reconfigurable remote node solution looks as a promising one. For short reach PON solutions, however, reconfigurability at the remote 
node may not be the way forward. Our study will help network operators and service providers to choose the suitable architecture for NGOA networks.

\section{Partially Flexible TWDM-PON}

In this section, we propose the architecture of a partially flexible TWDM-PON. In the last section, we found that a partially flexible architecture with a degree of multicasting as two and a switching element could give most of the advantages of flexibility. In this section, we propose such a design of a partially flexible TWDM-PON. At RN1, we use a combination of WSSs and AWGs. We call this architecture as the wavelength switched TWDM-PON. First, we discuss the system aspects and the advantages of the wavelength switched TWDM-PON, and then we compare cost, reach and power consumption of the wavelength switched TWDM-PON with other TWDM-PON architectures.

\subsection{System Aspects and Advantages}

Figure 8 shows the architecture of the wavelength switched TWDM-PON. At RN1, we assume a cascaded configuration of WSSs and AWGs. To compensate for the insertion loss of a WSS, we use EDFAs based reach extenders (RE). Two 1:K WSSs are used, for downstream and upstream, respectively. The WSSs are used together with 1:M cyclic athermalized AWGs. The AWGs are of an M-skip-0 configuration. Both AWGs and WSSs are of a $50 \mathrm{GHz}$ configuration. For a 40-channel configuration at the OLT, we use 1:4 WSS and 1:5 AWG. On an average, this configuration allows two wavelength channels at each output port of the AWG. This wavelength allocation, however, can be rearranged with a changing traffic requirement. As we discussed before, the traffic in a long-reach PON is bursty and thus there is a significant amount of energy saving that can be accrued due to re-routing wavelengths. Re-routing capacity leads to an improvement in the PON performance, minimizes wavelength consumption, and saves appreciable amount of energy. This on-demand re-routing can be easily implemented in the considered architecture. For example, on a high traffic demand from the users behind an AWG, all 40 channels can be fed to the AWG. This leads to about 8 channels per output port of the AWG. Similarly, when the demand from a TDM-PON is low, one of the two wavelengths can be switched off leading to energy efficiency.

Furthermore, the considered architecture can be made more energy efficient by assuming special functionality, e.g., broadcasting or selective multicasting at the WSS. 
In the next section, we propose an energy saving scheme, which assumes WSSs capable of multicasting wavelengths selectively. This, however, requires advanced WSSs with a higher cost. In addition, multicasting increases the insertion loss of the wavelength that is multicasted. For example, a wavelength that is multicasted to two groups will experience an additional insertion loss of $3 \mathrm{~dB}$.

The considered architecture has several additional advantages. For example, it helps operators to plan their network easily. An important aspect of network planning is to adapt the network to the requirements. There are many divergences in the requirements due to different scenarios, like urban and rural. For example, an urban scenario requires a shorter reach and a higher splitting ratio whereas a rural scenario requires a longer reach and a smaller splitting ratio. Thus, the power splitters with different splitting ratios are required. Moreover, the power splitters with different splitting ratios also require wavelengths to be distributed differentially among the power splitters to ensure the same bandwidth delivery per customer. This differential wavelength feed can easily be routed by WSSs. The differential wavelength feed can also be used to provision narrowband services. For example, should there be many users behind a power splitter requiring a narrowband service, a fewer number of wavelengths can be fed to a power splitter. Thus, the ability of WSSs to route wavelengths at RN2 dynamically will ease the network planning according to the scenario and requirements.

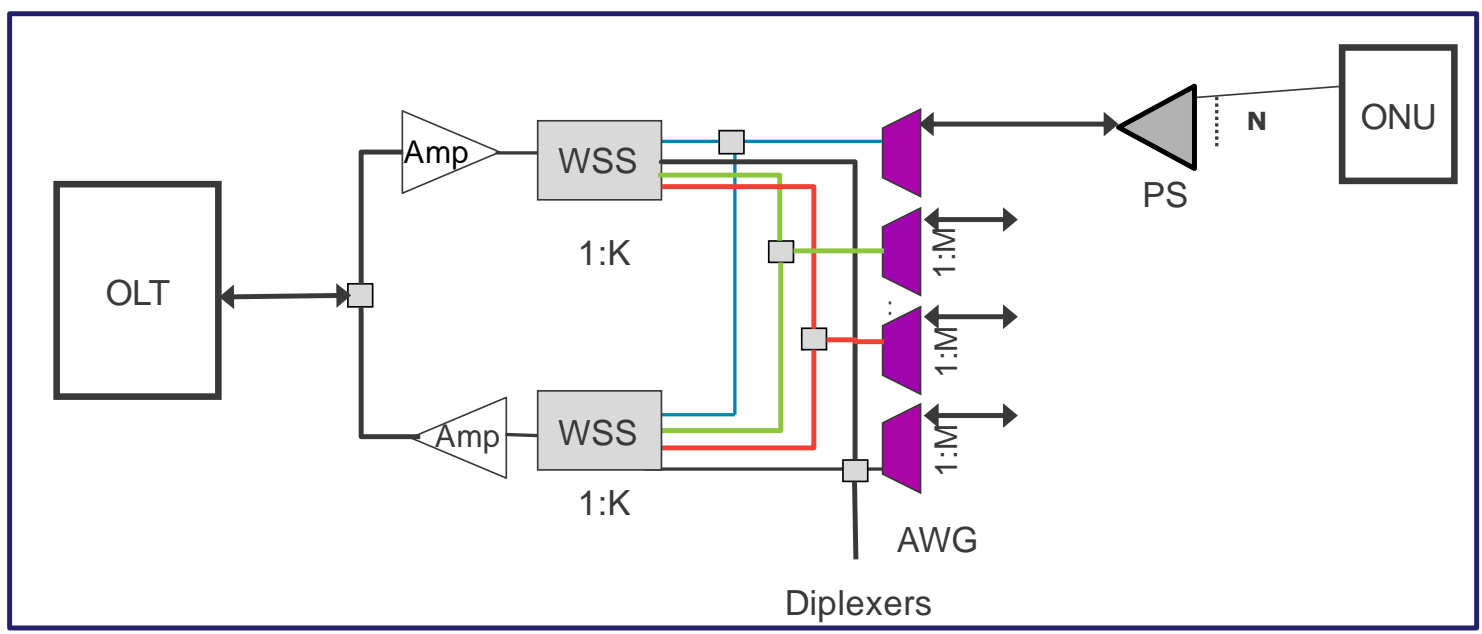

Figure 8: Wavelength Switched TWDM-PON architecture

\subsection{Evaluation of Wavelength Switched TWDM-PON}

In this section, we evaluate reach, cost and power consumption of the 40 and 80 channels wavelength switched TWDM-PON, and compare it to the wavelength selected and wavelength split TWDM-PON. For page space efficiency, wherever there is a 
difference between the configurations with 40 and 80 channels, we denote numbers corresponding to 80 channels in \{\} .

\subsubsection{Reach, power consumption and cost calculations for a wavelength switched TWDM-PON}

We show the calculations of reach, power consumption and cost for wavelength switched TWDM-PON. We use 1:4 WSS and 1:5 \{1:10\} AWG in RN1, and 1:32 power splitter in RN2.

Reach: The insertion Loss (IL) of the above configuration is the sum of the IL for OLT, RN1, RN2, ONU, end-of-life (EOL) and connectors. The IL for the OLT is due to a C/L band-splitter $(1.5 \mathrm{~dB})$ and two \{three\} diplexers $(3\{4.5\} \mathrm{dB})$. 1:4 WSS (5.5 dB), 1:5 $\{1: 10\}$ AWG $(4 \mathrm{~dB})$, and two C/L band-splitters $(3 \mathrm{~dB})$ lead to the IL in RN1. The IL at $\mathrm{RN} 2$ is due to the use of 1:32 $(17.5 \mathrm{~dB})$ power splitter. At the ONU, the IL is due to a $\mathrm{C} / \mathrm{L}$ band splitter. We assume an EOL and connector IL of $5.7 \mathrm{~dB}$. This leads to the total IL of $41.7\{43.2\} \mathrm{dB}$, as shown in the following equations:

$$
\begin{aligned}
& I L_{O L T}=I L_{\text {BandSplitters }}+2\{3\} \times I L_{\text {Diplexers }}=1.5 d B+2\{3\} \times 1.5 d B=4.5\{6\} d B \\
& I L_{R N 1}=I L_{W S S}+I L_{A W G}+2 \times I L_{\text {BandSplitter }} \\
& =5.5 d B+4 d B+2 \times 1.5 d B=12.5 d B \\
& I L_{R N 2}=I L_{\text {PowerSplitter }}=17.5 d B \\
& I L_{O N U}=I L_{\text {BandSplitter }}=1.5 d B \\
& I L_{E O L}+I L_{\text {Connectors }}=5.7 d B \\
& I L_{\text {Total }}=I L_{O L T}+I L_{R N 1}+I L_{R N 2}+I L_{O N U}+I L_{E O L}+I L_{\text {Connectors }}=41.7\{43.2\} d B
\end{aligned}
$$

The $10 \mathrm{G}$ transmitter at the OLT is assumed with a power level of $6 \mathrm{dBm}$, and the $10 \mathrm{G}$ avalanche photo-diode (APD) receiver with forward error correction (FEC) is assumed with a sensitivity of $-30 \mathrm{dBm}$. Use of booster $(4\{2.5\} \mathrm{dB})$ and $\mathrm{RE}(20 \mathrm{~dB})$ leads to a total fiber budget of $18.3\{15.3\} \mathrm{dB}$, which gives a reach of $54\{45\} \mathrm{km}$. Following equations show the reach calculations:

$$
\begin{aligned}
& \text { Re } a c h=\frac{\text { FiberBudget }}{\text { FiberLoss }}=\frac{T X-R X(\text { withFEC })+\text { Booster }+R E-I L_{\text {Total }}}{0.34 d B / \mathrm{km}} \\
& =\frac{6 \mathrm{dBm}-(-30 \mathrm{dBm})+4\{2.5\} \mathrm{dB}+20 \mathrm{~dB}-41.7\{43.2\} \mathrm{dB}}{0.34 \mathrm{~dB} / \mathrm{km}}=54\{45\} \mathrm{km}
\end{aligned}
$$


Note that the booster gain is assumed such that the total power in the fiber does not exceed $21 \mathrm{dBm}$ for the laser class $1 \mathrm{M}$ safety considerations. This restricts the total power in one channel to be below $5.5\{2.5\} \mathrm{dBm}$.

Power Consumption and Cost: Table 1 gives the cost and the power consumption of the OLT, RN1, RN2 and ONU, and the floor space for the OLT. The cost and power consumption values for various components and the floor space are from [40]. The cost values are normalized with the cost of a GPON ONU. The OLT is assumed with a shelf capacity of 20 slots, where two slots are reserved for uplink. The shelf includes mechanics, backplane, power supply (redundant), management and a layer 2 switch (modular). The layer 2 switch capacity per shelf can be incremented in 100 Gbps steps.

Table 1. Cost and power consumption of system concepts of a $40\{80\}$ channel wavelength switched TWDM-PON

\begin{tabular}{|c|c|c|c|c|c|}
\hline \multicolumn{3}{|c|}{ OLT } & \multirow{2}{*}{$\begin{array}{c}\text { RN1 } \\
\text { AWG }(1: 5\{10\}):\end{array}$} & \multirow{2}{*}{$\begin{array}{c}\text { RN2 } \\
\text { Power Splitter }\end{array}$} & \multirow{2}{*}{$\begin{array}{c}\text { ONU } \\
\text { CPE Mechanics: }\end{array}$} \\
\hline $10 \times 10$ G TRX & Shelf Port Cards: & Shelf Space: & & & \\
\hline Array TDMA: & Cost: & Cost: & Cost: & 1:32: & Cost: \\
\hline Cost: & $4\{8\} \times 10$ & $12\{22\}$ slots & 0.3 (per trib port) & Cost: & 0.6 \\
\hline $4\{8\} \times 26.26$ & $\times 10 \mathrm{Gbps}$ & $\times 5.56 /$ slot & & 6.6 & Power: \\
\hline Power: & $\times 0.1 / \mathrm{Gbps}$ & Power: & WSS (1:4): & & $3 \mathrm{~W}$ \\
\hline $4\{8\} \times 20 W$ & (port aggregator) & $12\{22\}$ slots & Cost: & & \\
\hline & $+6\{11\} \times 0.8$ & $\times 5 \mathrm{~W} / \mathrm{slot}$ & $2 \times 104$ & & 10 G TDMA \\
\hline MAC: & (mechanics for 12 & & Power: & & TRX (APD): \\
\hline Cost: & $\{22\}$ slots) & L2 switching: & $2 \times 5.5 \mathrm{~W}$ & & Cost: \\
\hline $40\{80\} \times 2$ & Power: & Shelf switch: & & & 2.5 \\
\hline Power: & $4\{8\} \times 10$ & $4\{8\} \times 10$ & Diplexer: & & Power: \\
\hline $40\{80\} \times 1 W$ & $\times 10 \mathrm{Gbps}$ & $\times 10$ Gbps $\times 18$ & Cost: & & $2.5 \mathrm{~W}$ \\
\hline Diplexers: & $\begin{array}{l}\times 0.5 \mathrm{~W} / \mathrm{Gbps} \\
\text { (port aggregator) }\end{array}$ & $\begin{array}{l}\text { slots } / 12\{22\} \text { slots } \\
=600\{700\}\end{array}$ & $5 \times 0.3$ & & \\
\hline Cost: & $+6\{11\} \times 10$ & Gbps; & Reach Extender: & & \\
\hline $7\{15\} \times 0.3$ & (base for $12\{22\}$ & Cards per shelf: & Cost: & & \\
\hline Shelf Space: & slots) & $1.5\{0.8\}$ & $2 \times 20$ & & \\
\hline $2\{4\}$ slots & Shelfspace: & Cost: & Power: & & \\
\hline & $4\{8\} \times 2$ slots & $\begin{array}{l}600\{700\} \text { Gbps } \times \\
0.1 / \mathrm{Gbps} / 1.5\{0.8\}\end{array}$ & $2 \times 50 \mathrm{~W}$ & & \\
\hline & Booster and Pre- & Power: & & & \\
\hline & Amp: & $600\{700\}$ Gbps $\times$ & & & \\
\hline & Cost: & $1 \mathrm{~W} / \mathrm{Gbps} / 1.5\{0.8\}$ & & & \\
\hline & $2 \times 15$ & & & & \\
\hline & Power: & & & & \\
\hline & $2 \times 12 \mathrm{~W}$ & & & & \\
\hline & Shelf space: & & & & \\
\hline & 2 slot & & & & \\
\hline
\end{tabular}

\subsection{Comparison of different TWDM-PON variants}

In this section, we compare different TWDM-PON variants. The wavelength selected TWDM-PON has a power splitter and a RE at RN1. It requires a RE to support a high customer fan out and a reach of $49\{30\} \mathrm{km}$. Furthermore, the number of customers in the wavelength selected TWDM-PON is assumed as 560/1024 because not all splitting ratios are possible for a power splitter. For other configurations, the number of customers is 640/1280. The wavelength split TWDM-PON has an AWG at RN1, and can support a pure passive reach of $27\{16\} \mathrm{km}$. With a RE, however, it can have an 
additional reach of $59 \mathrm{~km}$ to meet the NGOA requirements. Figure 9 compares the cost, power consumption (PC) and the reach of various TWDM-PON configurations. When comparing wavelength switched TWDM-PON with the other variants, first, we show that the additional cost and power consumption of using a WSS at RN1 is negligible as compared to the completely passive configuration, because many users (640 or 1280) share the cost and power consumption of RN1. Secondly, the use of amplifiers in RN1 compensates the insertion loss of WSSs and gives a long reach of $54\{45\} \mathrm{km}$. Besides, as mentioned before, WSS leads to several other advantages, which will further bring down the operational expenditures. This helps us to conclude that the use of WSSs will accrue significant advantages while adding an insignificant component cost and power consumption per user.

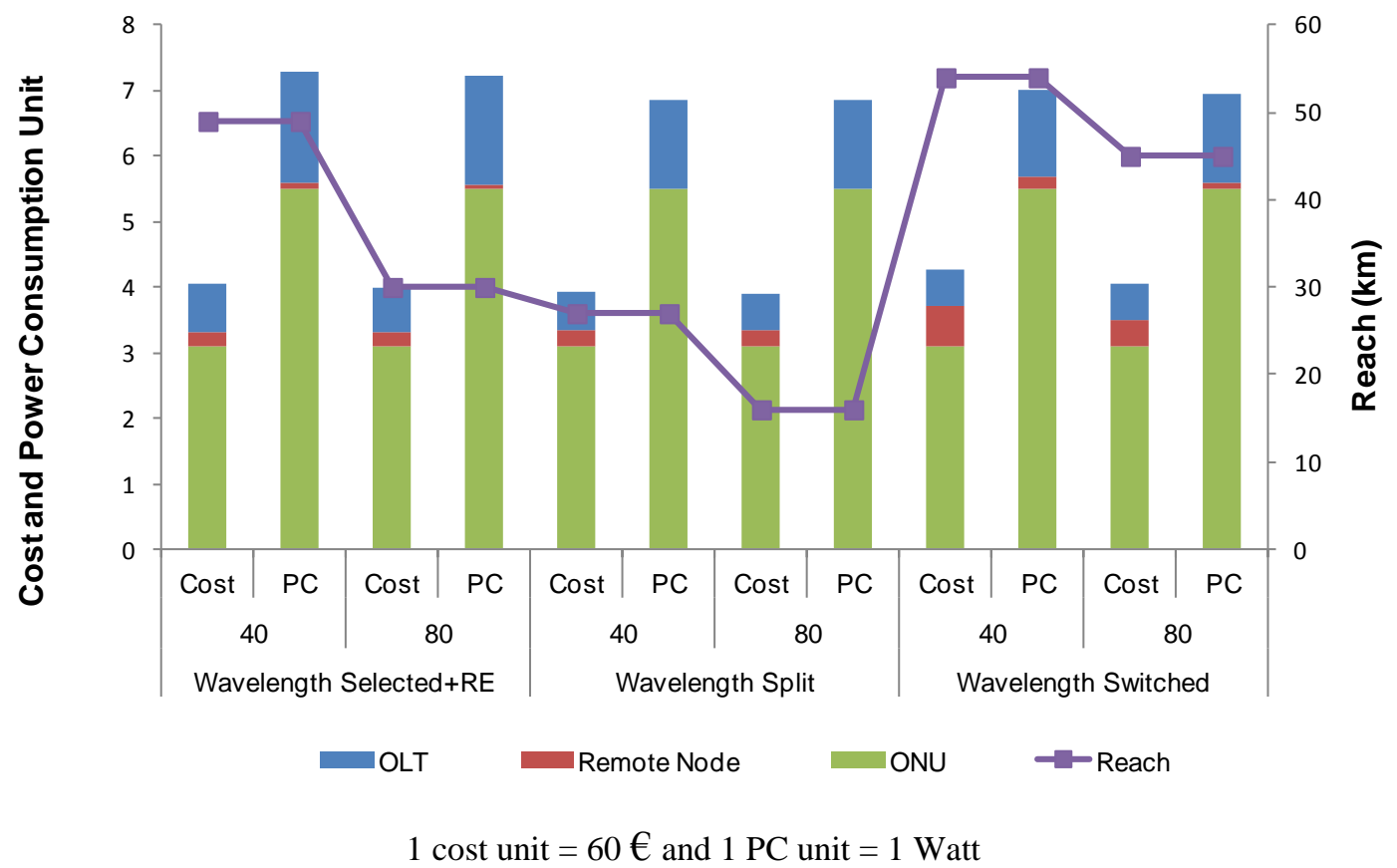

Figure 9: Cost, Power Consumption and Reach comparison of various TWDM-PON variants

\subsection{Conclusions}

In this section, we have proposed a novel architecture for a TWDM- PON using WSS as the key component. Through a detailed reach and cost evaluation, we have shown that it provides the best combination of reach, flexibility, security and cost among all the existing TWDM-PON technologies. 


\section{Energy efficient DBA algorithm for wavelength switched TWDM -PON}

In this section, we discuss the proposed DBA scheme, and analyze the energy savings possible because of that. As discussed in the last section, WSSs have a great potential to save energy because of their virtue to split wavelengths selectively. This selective splitting of wavelengths enables selective multicasting, and is utilized in the proposed algorithm. The basic idea of the proposed algorithm is to optimize the grouping of users on wavelengths, which leads to minimal use of wavelengths and save energy. The number of ONUs that can be grouped on a wavelength is limited by either the data rate or the distances of the ONUs from the OLT. For example, should the offered load of the ONUs is low, many nearby ONUs can be served using a single wavelength. As the load increases, however, not all previously grouped ONUs can be served by the same wavelength, and the group of ONUs has to be allocated an additional wavelength. For distant ONUs, the wavelength cannot undergo a high splitting, as otherwise the fiber budget considerations cannot be met.

Figure 10 shows the grouping of ONUs on a wavelength based on their distances from the OLT. For example, let us assume that on the first port of the AWGs, nearby ONUs are connected over a power splitter. Since, the distances of these ONUs from the OLT is short, the power budget is less constrained and thus these groups of ONUs can be served on a single wavelength if the offered load of the ONUs is low. Nevertheless, when the load of the ONUs becomes high, the assured throughput of every ONU cannot be met. Hence, as soon as the load of the ONUs increases, these ONUs have to be allocated additional wavelengths. For the ONUs that are far, a higher splitting of one wavelength does not meet the power budget requirements, and hence distant ONUs require more number of wavelengths even at a low load. This policy of grouping ONUs based on their distances, while still satisfying the data rate requirements of the ONUs, increase the number of customers served on the a wavelength and reduce the power consumption per customer. In this way, we minimize the use of transceivers and switch them off, leading to the savings in energy consumption. We call this approach as the data and distance based grouping (D\&DBG). 


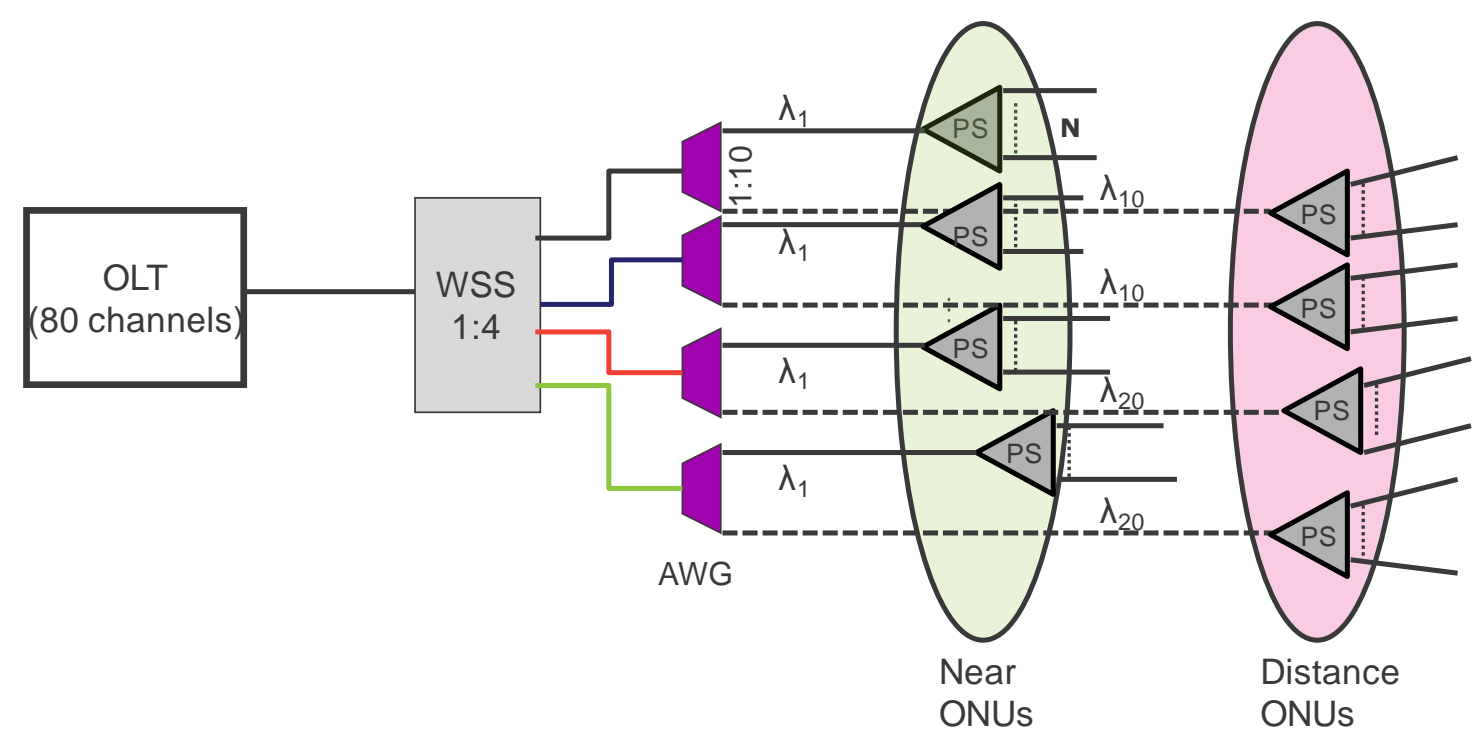

Figure 10: Illustrates the grouping of users on a wavelength based on their distances from the OLT.

For the analysis, we assume that the wavelengths could be switched off in the granularity of a transceiver array, and a switched off transceiver array also minimizes energy consumption due to shelf port cards, shelf space and layer 2 switching. Currently, the OLT rack design does not support individual powering off the slots; we assume, however, that in the near future such a design should be made possible to conserve energy. Moreover, we assume that the layer 2 switches of smaller capacity are individually connected to a TRXA, so that it is possible to turn them on/off according to the TRXA requirement. Figure 11 (a) shows the energy consumption of the OLT with the number of wavelengths used. The energy consumption variation is a step function as the wavelengths can be turned off in the granularity of the number of wavelengths in a transceiver array. Nevertheless, to harness better energy efficiency gains, the individual shut down of each channel should be the primary design goals of these multi-channels arrays.

Figure 11 (b) shows the energy consumption of the OLT when the ONUs are grouped on the same wavelength according to the distances still satisfying the data rate of the users. For this result, the ONUs are assumed to be distributed uniformly between 20 and $100 \mathrm{~km}$. The $100 \%$ is the energy consumption when all transceivers are on. Without the use of D\&DBG approach, in normal mode, there is already energy saving of about $50 \%$ at the low data rate because of the flexibility of the architecture. With D\&DBG approach, the energy consumption is further reduced to only $40 \%$. The savings at OLT also save power for cooling, and reduce operational expenditure of the network provider. The power for cooling is typically the double of the system power used. 


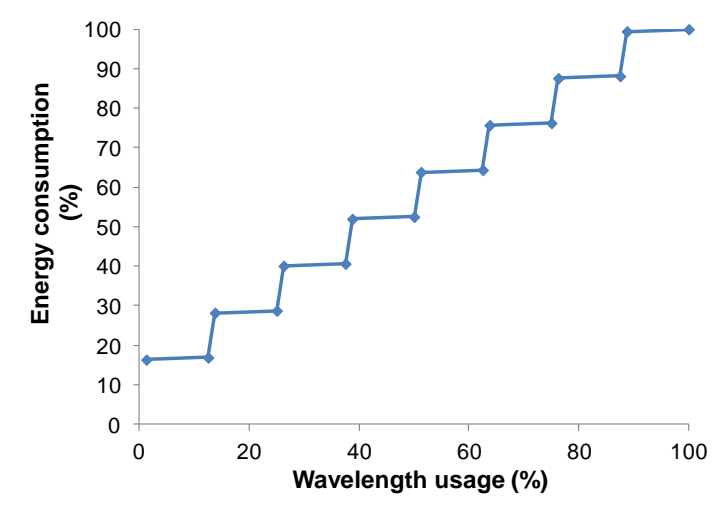

(a)

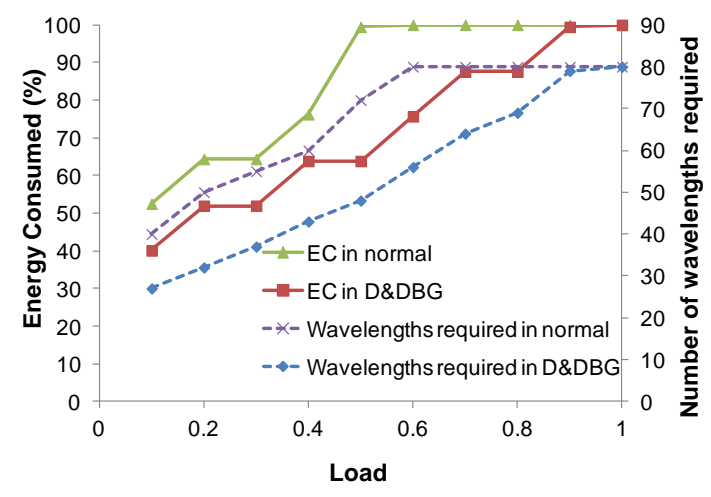

(b)

Figure 11: a) Variation of energy consumption with wavelength usage at the OLT b) energy consumption (EC) and the number of wavelengths required in normal and data and distance based grouping (D\&DBG) approach: energy consumption shown using vertical right axis and the number of wavelengths required shown using dashed lines and vertical left axis

\section{Conclusions}

Hybrid TDMA/WDMA (TWDM) passive optical networks (PON) have emerged as one of the important next-generation optical access (NGOA) solutions. These solutions can further be complemented by adding flexibility, which reflects the dynamicity of resource sharing. There are two options to provide flexibility in the network: using power splitters, and using wavelength routing switches, e.g., wavelength selective switches (WSS). Power splitters broadcast wavelengths to users, providing multicasting gains, which increase wavelength utilization and energy savings. On the other hand, WSSs can route traffic to the more congested part of the network, furnishing the gains of load balancing. The flexibility due to WSSs is referred as switching flexibility. From exhaustive simulations, we conclude that a partially flexible network can already provide enough multicasting gains compared to a fully flexible network. Moreover, a partially flexible network further benefits from the switching flexibility, particularly in the scenario where the traffic is highly bursty. Further, we proposed a partially flexible solution, based on a cascaded configuration of WSS and arrayed waveguide grating (AWG), which is called as wavelength switched TWDM-PON. The wavelength switched TWDM-PON accrues flexibility advantages without adding significant cost, power consumption and insertion loss. Finally, we also proposed an energy saving scheme for wavelength switched TWDM-PON that has the potential of saving up to $60 \%$ energy at the OLT. 


\section{Acknowledgements}

The research leading to these results has received funding from the European Community's Seventh Framework Programme (FP7/2007-2013) under grant agreement $\mathrm{n}^{\circ} 249025$ (ICT-OASE), and grant agreement $\mathrm{n}^{\circ} 257740$ (Network of Excellence TREND).

\section{References}

[1] ITU-T Recs. G.983, BPON; G.984, GPON.

[2] IEEE Stds. 802.3ah, Ethernet First Mile (EFM); 802.3av, 10GEPON.

[3] Global Passive Optical Network Equipment Market 2010-2014 [Online] Available at: http://www.researchandmarkets.com/research/267627/global_passive_opt

[4] L. Kazovsky, S. W. Wong, S. H. Yen, V. Gudla, P. Afshar, and D. Larrabeiti, Next-Generation Optical Access Networks: Goals, Challenges and Research Opportunities, Acreo/KTH Seminar, Stockholm, Sweden, August 2010.

[5] 249025-ICT OASE Project, Requirements for European Next-Generation Optical Access Networks, D2.1.

[6] Y. Luo, X. Zhou, F. Effenberger, X. Yan, G. Peng, Y. Qian, and Y. Ma, Time and Wavelength Division Multiplexed Passive Optical Network (TWDM-PON) for Next Generation PON Stage 2 (NG-PON2), Journal of Lightwave Technology 31 (4) (2013) 587 - 593.

[7] A. Dixit, B. Lannoo, G. Das, D. Colle, M. Pickavet, and P. Demeester, Evaluation of flexibility in hybrid WDM/TDM PONs, in Proc. IEEE International Symposium on Access Spaces (ISAS), Yokohama, Japan, June 2011.

[8] A. Dixit, B. Lannoo, G. Das, D. Colle, M. Pickavet, and P. Demeester, Flexibility Evaluation of Hybrid WDM/TDM PONs, IEEE ANTS 2011, Bangalore, India, Dec. 18-21, 2011.

[9] M. Ruffini, D. Mehta, B. O"Sullivan, L. Quesada, L. Doyle, and D. B. Payne, Deployment strategies for protected long-reach PON, IEEE/OSA Journal of Optical Communication Network 4 (2) (2012) 118 - 129.

[10]R. D. Feldman, Crosstalk and loss in wavelength division multiplexed systems employing spectral slicing, Journal of Lightwave Technology 15 (11) (1997) 1823-1831.

[11]P. Healey, P. Townsend, C. Ford, L. Johnston, P. Townley, I. Lealman, L. Rivers, S. Perrin, and R. Moore, Spectral slicing WDM-PON using wavelength-seeded reflective SOAs, Electron. Letters 37 (19) (2001) 1181-1182.

[12] S.-J. Park, C.-H. Lee, K.-T. Jeong, H.-J. Park, J.-G. Ahn, and K.-H. Song, Fiber-to-the-home services based on wavelength-division multiplexing passive optical network, Journal of Lightwave Technology 22 (11) (2004) 2582-2591.

[13]D. J. Shin, D. K. Jung, H. S. Shin, J. W. Kwon, S. Hwang, Y. Oh, and C. Shim, Hybrid WDM/TDMPON with wavelength-selection-free transmitters, Journal of Lightwave Technology 23 (1) (2005) 187-195. 
[14]L. Y. Chan, C. K. Chan, D. T. K. Tong, F. Tong, and L. K. Chen, Upstream traffic transmitter using injection-locked Fabry-Pérot laser diode as modulator for WDM access networks, Electronics Letters 38 (1) (2002) 43-45.

[15] N. J. Frigo, P. P. Iannone, P. D. Magill, T. E. Darcie, M. M. Downs, B. N. Desai, U. Koren, T. L. Koch, C. Dragone, H. M. Presby, and G. E. Bodeep, A wavelength-division multiplexed passive optical network with cost-shared components, IEEE Photonics Technology Letters 6 (11) (1994) 1365-1367.

[16]L. Altwegg, A. Azizi, P. Vogel, Y.Wang, and F.Wyler, LOCNET: A fiber in the loop system with no light source at the subscriber end, Journal of Lightwave Technology 12 (3) (1994) 535-540.

[17] K. Iwatsuki, J.-I. Kani, H. Suzuki, and M. Fujiwara, Access and metro networks based on WDM technologies, Journal of Lightwave Technology 22 (11) (2004) 2623-2630.

[18] G. Talli and P. D. Townsend, Feasibility demonstration of $100 \mathrm{~km}$ reach DWDM Super-PON with upstream bit rates of $2.5 \mathrm{~Gb} / \mathrm{s}$ and $10 \mathrm{~Gb} / \mathrm{s}$, in Proc. Optical Fiber Communication Conf., Glasgow, U.K., 2005, Paper OFI1.

[19]A. Dixit, B. Lannoo, D. Colle, M. Pickavet, and P. Demeester, Wavelength switched hybrid WDM/TDMA PON: A flexible next-generation optical access solution,, in Proc. ICTON 2012, Coventry, UK, July 2012.

[20] J. Kani, S. Shimazu, N. Yoshimoto, and H. Hadama, Energy-efficient optical access networks: issues and technologies, IEEE Communications Magazine 51 (2) (2013) S22 - S26.

[21]G. Das, B. Lannoo, D. Colle, M. Pickavet, and P. Demeester,, A Hybrid WDM/TDM PON Architecture Using Wavelength Selective Switches, in Proc. IEEE ANTS, Mumbai, India, Dec. 16$18,2010$.

[22] G. Das, B. Lannoo, A. Dixit, D. Colle, M. Pickavet, and P. Demeester, Flexible hybrid WDM/TDM PON architectures using wavelength selective switches, Optical Switching and Networking 9 (2) (2012) 156-169.

[23]C. Bock, J. Prat, and S. D. Walker, Hybrid WDM/TDM PON Using the AWG FSR and Featuring Centralized Light Generation and Dynamic Bandwidth Allocation, Journal of Lightwave Technology 23 (12) (2005) 3981-3988.

[24] K. Grobe, M. Roppelt, A. Autenrieth, J. P. Elbers, and M. Eiselt, Cost and Energy Consumption Analysis of Advanced WDM-PONs, IEEE Communications Magazine 49 (2) (2011) s25 - s32.

[25]N. C. Tran, H. D. Jung, C. Okonkwo, E. Tangdiongga, and T. Koonen, A 10 Gb/s PassiveComponents-based WDM-TDM Reconfigurable Optical Access Network Architecture, in Proc. Optical Fiber Communication Conference and Exposition (OFC/NFOEC), 2011 and the National Fiber Optic Engineers Conference, Los Angeles, U.S.A, March 6-10, 2011.

[26] N.C. Tran, H.D. Jung, C. Okonkwo, E. Tangdiongga, and T. Koonen, ARON: A SOA Array-based WDM-TDM Reconfigurable Optical Access Network, Future Network \& Mobile Summit 2010, pp. 1-7 (2010).

[27]P. J. Urban, B. Huiszoon, R. Roy, M. M. de Laat, F. M. Huijskens, E. J. Klein, G. D. Khoe, A. M. J. T. Koonen, and H. de Waardt, High-Bit-Rate Dynamically Reconfigurable WDM-TDM Access Network, Journal of Optical Communications and Networking 1 (2) (2009) A143 - A158. 
[28] S. H. Yen, M. Tendulkar, J. R. Jameson, S. Yamashita, Y. Nishi, O. Solgaard, and L. G. Kazovsky, Quasi-passive and reconfigurable node for optical access network, Lasers and Electro-Optics (CLEO) and Quantum Electronics and Laser Science Conference (QELS), San Jose, CA, USA, May 2010 .

[29]B. Lannoo, G. Das, A. Dixit, D. Colle, M. Pickavet, and P. Demeester, "Novel hybrid WDM/TDM PON architectures to manage flexibility in optical access networks," Telecommunications System Journal, in press.

[30] A. Dixit, B. Lannoo, G. Das, D. Colle, M. Pickavet, and P. Demeester, Evaluation of ONU Power Saving Modes in Next Generation Optical Access Networks, in Proc. ECOC 2012, Amsterdam, Netherlands, Sep. 2012.

[31] A. Dixit, B. Lannoo, D. Colle, M. Pickavet, and P. Demeester, ONU Power Saving Modes in Next Generation Optical Access Networks: Progress, Efficiency and Challenges, Optics express 20 (26) (2012) B52-B63.

[32]A. Dixit, B. Lannoo, G. Das, D. Colle, M. Pickavet, and P. Demeester, Dynamic Bandwidth Allocation with SLA Awareness for QoS in Ethernet Passive Optical Networks, Journal of Optical Communications and Networking 5 (3) (2013) 240 - 253.

[33] W. Willinger, S. M. Taqqu, R. Sherman, and D. V. Wilson, Self-similarity through high variability: statistical analysis of Ethernet LAN traffic at the source level, IEEE/ACM Transactions on Networking 5 (1) (1997) 71-86.

[34] W.E Leland, S. M. Taqqu, W. Willinger and D. V. Wilson, On the self-similar nature of Ethernet traffic, IEEE/ACM Transactions on Networking 2 (1) (1994) 1-15.

[35] G. Das, B. Lannoo, D. Jung, T. Koonen, D. Colle, M. Pickavet, and P. Demeester, A New Architecture and MAC Protocol for Fully Flexible Hybrid WDM/TDM PON, Proc. of ECOC 2009, Vienna, Austria, Sep. 20 - 24, 2009, paper P6.28.

[36] A. Rohit, A. Albores-Mejia, N. Calabretta, X. J. M. Leijtens, D. J. Robbins, M. K. Smit and K. A. Williams, Fast Remotely Reconfigurable Wavelength Selective Switch, in Proc. OFC/NFOEC 2011, OTuM.1.

[37] J. Zhang, and N. Ansari, Scheduling Hybrid WDM/TDM Passive Optical Networks with Nonzero Laser Tuning Time, IEEE/ACM Transactions on Networking 19 (4) (2011) 1014 - 1027.

[38] B. Lannoo, L. Verslegers, D. Colle, M. Pickavet, M. Gagnaire, and P. Demeester, Analytical Model for the IPACT Dynamic Bandwidth Allocation Algorithm for EPONs, Journal of Optical Networking 6 (6) (2007) 677-688.

[39]Flexible Wavelength Selective Switches (WSS) for Advanced Network Architectures and for Advanced Network Architectures and Modulation Formats [Online] Available at: http://www.ofcnfoec.org/osa.ofc/media/Default/PDF/2009/09-Poole.pdf

[40]249025-ICT OASE Project, D4.2.1: Technical Assessment and Comparison of Next-Generation Optical Access System Concepts, Oct. 2011. 